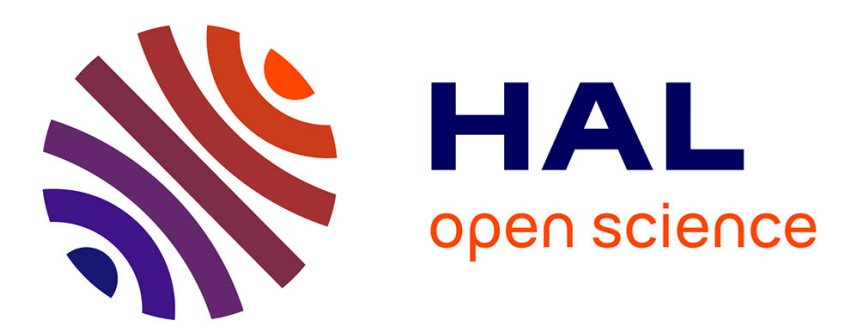

\title{
Numerical study on hydrodynamic and quasi-neutral approximations for collisionless two-species plasmas
}

\author{
Simon Labrunie, José A. Carrillo, Pierre Bertrand
}

\section{To cite this version:}

Simon Labrunie, José A. Carrillo, Pierre Bertrand. Numerical study on hydrodynamic and quasineutral approximations for collisionless two-species plasmas. Journal of Computational Physics, 2004, 200 (1), pp.267-298. 10.1016/j.jcp.2004.04.020 . hal-00141789

\section{HAL Id: hal-00141789 \\ https://hal.science/hal-00141789}

Submitted on 16 Apr 2007

HAL is a multi-disciplinary open access archive for the deposit and dissemination of scientific research documents, whether they are published or not. The documents may come from teaching and research institutions in France or abroad, or from public or private research centers.
L'archive ouverte pluridisciplinaire HAL, est destinée au dépôt et à la diffusion de documents scientifiques de niveau recherche, publiés ou non, émanant des établissements d'enseignement et de recherche français ou étrangers, des laboratoires publics ou privés. 


\title{
Numerical study on hydrodynamic and quasi-neutral approximations for collisionless two-species plasmas
}

\author{
Simon Labrunie $^{\mathrm{a}, *}$, José A. Carrillo ${ }^{\mathrm{b}}$, Pierre Bertrand ${ }^{\mathrm{c}}$ \\ ${ }^{a}$ Institut Élie Cartan (Mathématiques), Université Henri Poincaré Nancy 1 et \\ INRIA (projet CALVI), 54506 Vandoeuvre-lès-Nancy, France \\ ${ }^{\mathrm{b}}$ Departament de Matemàtiques - ICREA, Universitat Autònoma de Barcelona, \\ 08193 Bellaterra, Spain \\ ${ }^{\mathrm{c}}$ Laboratoire de physique des milieux ionisés, Université Henri Poincaré Nancy 1, \\ 54506 Vandouvre-lès-Nancy, France
}

\begin{abstract}
We present a numerical study aimed at assessing the validity of certain approximations, chiefly the fluid and quasi-neutral ones, generally used in the theoretical and computational study of fusion plasmas. The impact of numerical artifacts has been minimised by using the very precise Weighted Essentially Non-Oscillatory (WENO) method, which can be applied to both kinetic and fluid simulations.

The fluid modelling of plasmas appears adequate even when collisions are negligible, provided certain relationships hold between the characteristic speed of the phenomenon under consideration and the thermal velocities of the species which make up the plasma. The breakdown of this behaviour is probably caused by Landau damping; it appears linked with filamentation, which suggests that the magnitude of the departure from Maxwellian equilibrium may not be the one and only measurement of non-fluid behaviour. As for quasi-neutrality, our study suggests that the generally accepted criteria should be used with great care, according to the degree of neutrality expected.
\end{abstract}

Key words: computational plasma physics, Vlasov-Poisson system, fluid modelling of plasmas, quasi-neutrality, WENO schemes

PACS: 52.65.-y, 52.65.Ff, 52.65.Kj, 52.27.Aj, 02.70.Bf

* Corresponding author.

Email addresses: labrunie@iecn.u-nancy.fr (Simon Labrunie), carrillo@mat.uab.es (José A. Carrillo), bertrand@lpmi.u-nancy.fr (Pierre Bertrand). 


\section{Introduction}

Collective effects play an important role in low-density, high-temperature plasmas. This is especially true in fusion plasmas. A full kinetic description needs to solve the Vlasov-Maxwell equations. Even in the simplified 1D VlasovPoisson system, the complexity of this model invites us to study simpler ones, among which the hydrodynamic or fluid description plays a central role. Both from a theoretical and a computational point of view, the use of the fluid approach is considerably simpler than the Vlasov one.

Of course, if Landau damping is present, the hydrodynamic model is unable to model the resonant wave-particle interactions. On the other hand if kinetic effects can be neglected, i.e. the distribution function remains close to the local thermodynamic equilibrium, fluid equations give a good description of plasma turbulence with cross fertilisation between plasma physics and fluid mechanics (Kelvin-Helmoltz, convective cells, intermittency, zonal flows,...).

But in the real world, the choice between the two models - kinetic and fluid - is not so obvious. This is particularly true in studying turbulence and anomalous transport in tokamak. The computation of turbulent thermal diffusivities in fusion plasmas is of prime importance since the energy confinement time is determined by these transport coefficients. During recent years, ion turbulence in tokamaks has been intensively studied both with fluid (see for instance [13]) and kinetic simulations (see References [4,5] using particle-in-cell (PIC) simulations and [6,7] using Vlasov simulations). Although more accurate, the kinetic calculation of turbulent transport is much more demanding in computer resources than fluid simulations. As already mentioned, a key issue is the resonant interaction between waves and particles, which has to be accurately described. These resonant interactions probably play an important role, at least close to the instability threshold [8]. As a matter of fact the thermal diffusivity $\chi$ computed from fluid simulations exhibits an overestimate as compared to kinetic simulations: $\chi_{\mathrm{flu}} \simeq 2 \chi_{\text {kin }}$.

To address this point, it is not necessary to study such a complicated system as a tokamak plasma, and we can go back to much simpler academic models. First of all let us consider a collisionless, one-dimensional electron plasma in a fixed homogeneous neutralising background. It is well known that using the first three moments of the Vlasov-Poisson system and neglecting the heat flux allows us to recover the Euler-Poisson equations with the Lagrangian adiabaticity relation $\frac{\mathrm{d}}{\mathrm{d} t}\left(p n^{-3}\right)=0(p$ and $n$ being respectively pressure and electron density). This way of closing the hydrodynamic hierarchy needs physical arguments that are hard to demonstrate. Moreover a linearised version of the Euler-Poisson equations around an equilibrium yields the well-known long 
wavelength Bohm-Gross dispersion relation $\omega^{2}=\omega_{\mathrm{p}}^{2}+3 \mathrm{k}^{2} v_{\mathrm{th}}^{2}$ which can be obtained also from the linearised Vlasov equation in the long wavelength limit (negligible Landau damping).

On the other hand, the so called water bag model is not an approximation, but rather a subset of the Vlasov model (see for instance $[9,10]$ ). In such a model the electron distribution function $f_{e}$ is assigned a constant value $A$ inside some region of phase space plane bounded by two curves $v_{+}(t, x)$ and $v_{-}(t, x)$, and zero elsewhere. Assuming that $v_{+}$and $v_{-}$are single valued functions of $x$ at any time $t$, it can be easily shown that the heat flux is exactly zero, giving rigorously the Eulerian adiabatic law $\frac{\partial}{\partial t}\left(p n^{-3}\right)=\frac{\partial}{\partial x}\left(p n^{-3}\right)=0$.

Thus the water bag being characterised by a special class of initial conditions, is strictly equivalent to the Vlasov-Poisson system for all wavelengths and all degrees of nonlinearity. It is the unique example of an exact link between the Vlasov and hydrodynamic models, due to the analogy between the adiabatic laws. Notice that the pressureless gas (which is also described by the EulerPoisson equations without pressure, see (39) below) is simply a special instance of the water bag model in which the two contours $v_{+}(t, x)$ and $v_{-}(t, x)$ take on the same value.

Now, for any class of initial conditions but the water bag ones, and in the absence of collisions, there is no mechanism to ensure the relaxation of the distribution function towards some local thermodynamic equilibrium, allowing to close the hydrodynamic hierarchy obtained from the successive moments of the Vlasov equation.

The situation is more complicated for a two-species plasma (electron and mobile ions) such as the already mentioned tokamak plasma. Turbulence bearing usually low frequency modes, the electron fluid equations are closed using an isothermal approximation or described by some "Boltzmannian" density distribution, while the ions are assumed adiabatic; finally Poisson's equation is replaced by a quasi-neutrality approximation.

It is the purpose of this paper to compare these models, and check the pertinence of these fluid approximations with the full kinetic Vlasov description. Numerical codes are nowadays very precise tools to deal with and provide a reliable basis for numerical experiments with a high degree of accuracy. As already mentioned, there is no need to deal with too much complicated problems of magnetised fusion plasmas involving 3D complex geometries. Without loss of generality we can restrict to 1D two-species plasma and select a limited number of parameters. 
Thus, we shall consider the following simplified system as the object of this article. The plasma is totally ionised, i.e. made of electrons, with charge $-e$ and mass $m_{1}$, and ions with charge $Z e$ and mass $m_{2}$. (All quantities bearing the subscript 1 , resp. 2, will be relative to the electrons and the ions respectively.) It is one-dimensional and periodic: all quantities depend on only one space variable $x$, with a space period $L$, and all the velocities and the electric field are parallel to the $x$-axis. Global electrical neutrality is satisfied, i.e. the average electron density over one period is $Z$ times the average ion density. Finally, collisions between like or unlike particles are neglected, i.e. we observe the plasma for a time small compared to the collision period, and on a length scale somewhat larger than the Debye length.

\section{An overview of the physical models}

2.1 The mesoscopic description: two-species Vlasov-Poisson kinetic system

The distribution functions $f_{i}(t, x, v), i=1,2$, associated to electrons and ions satisfy the Vlasov equation

$$
\frac{\partial f_{i}}{\partial t}+v \frac{\partial f_{i}}{\partial x}+\mu_{i} E \frac{\partial f_{i}}{\partial v}=\frac{\partial f_{i}}{\partial t}-\left[\frac{v^{2}}{2}+\mu_{i} \phi, f_{i}\right]=0
$$

where $\mu_{i}$ denotes the signed charge/mass ratio, viz. $\mu_{1}=-e / m_{1}, \mu_{2}=Z e / m_{2}$; and $[\cdot, \cdot]$ is the Poisson bracket. They are coupled to the Poisson (or Gauss) equation for the electric field and potential $E(t, x)=-\frac{\partial \phi}{\partial x}$ :

$$
-\frac{\partial^{2} \phi}{\partial x^{2}}=\frac{\partial E}{\partial x}=\frac{e}{\varepsilon_{0}} \int_{-\infty}^{+\infty}\left(Z f_{2}-f_{1}\right) \mathrm{d} v
$$

The periodicity condition reads:

$$
f_{i}(t, x+L, v)=f_{i}(t, x, v), i=1,2, \quad E(t, x+L)=E(t, x) .
$$

Initial conditions have to be supplied. Without losing too much generality, we can assume them to be Maxwellian distributed, namely

$$
f_{i}(0, x, v)=n_{i}^{0}(x) \mathcal{M}_{\theta_{i}^{0}}\left(v-u_{i}^{0}(x)\right), \quad i=1,2,
$$


where

$$
\theta_{i}^{0}=\frac{k_{\mathrm{B}} T_{i}^{0}}{m_{i}}, \quad \mathcal{M}_{\theta}(v)=\frac{1}{\sqrt{2 \pi \theta}} \mathrm{e}^{-v^{2} / 2 \theta}
$$

and the density profiles $n_{i}^{0}(x)$ satisfy:

$$
\frac{1}{L} \int_{0}^{L} n_{1}^{0}(x) \mathrm{d} x=\frac{Z}{L} \int_{0}^{L} n_{2}^{0}(x) \mathrm{d} x \equiv \bar{n}, \quad n_{i}^{0}(x+L)=n_{i}^{0}(x), \quad i=1,2 .
$$

The mass conservation property of the Vlasov equation allows global electrical neutrality to be conserved with time, which causes the electric field to remain periodic in space, and this in turn extends to the distribution function.

Another important preserved global quantity is the total energy in the system given by

$$
W(t) \stackrel{\text { def }}{=} \sum_{i=1}^{2} m_{i} \int_{0}^{L} \int_{-\infty}^{+\infty} \frac{|v|^{2}}{2} f_{i}(t, x, v) \mathrm{d} v \mathrm{~d} x+\frac{\varepsilon_{0}}{2} \int_{0}^{L} E(t, x)^{2} \mathrm{~d} x
$$

for any $t \geq 0$, verifying formally $W^{\prime}(t)=0$ and then $W(t)=W(0)=W^{0}$. Let us finally remark that the two-species kinetic system has a lot of preserved quantities like any integral of functions of the distribution functions $f_{i}$. In particular it preserves the global "free energy" or relative entropy of the system:

$$
S(t) \stackrel{\text { def }}{=} \sum_{i=1}^{2} \int_{0}^{L} \int_{-\infty}^{+\infty} f_{i}(t, x, v) \ln f_{i}(t, x, v) \mathrm{d} v \mathrm{~d} x+W(t)
$$

for any $t \geq 0$, verifying formally $S^{\prime}(t)=0$ and then $S(t)=S(0)=S^{0}$.

\subsection{The macroscopic fluid description: two-species Euler-Poisson system}

For each species, we introduce the fluid quantities: density, current, kinematic energy density

$$
\left\{n_{i}, j_{i}, w_{i}\right\}(t, x)=\int_{-\infty}^{+\infty}\left\{1, v, \frac{|v|^{2}}{2}\right\} f_{i}(t, x, v) \mathrm{d} v
$$


and the fluid velocity $u_{i}=j_{i} / n_{i}$. Then, the kinematic pressure and heat flux are

$$
\begin{aligned}
& p_{i}(t, x)=\int_{-\infty}^{+\infty}\left(v-u_{i}(t, x)\right)^{2} f_{i}(t, x, v) \mathrm{d} v, \\
& K_{i}(t, x)=\frac{1}{2} \int_{-\infty}^{+\infty}\left(v-u_{i}(t, x)\right)^{3} f_{i}(t, x, v) \mathrm{d} v .
\end{aligned}
$$

Energy density and pressure are linked by the relation: $2 w_{i}=n_{i} u_{i}^{2}+p_{i}$; in other words, the so-called "adiabatic exponent" (cf. (20) below) is necessarily equal to 3. This stems from the one-dimensional character of the velocity space. Finally, we define the kinematic temperature as: $\theta_{i}=p_{i} / n_{i}$, i.e. $k_{\mathrm{B}} / m_{i}$ times the usual temperature in Kelvin.

The fluid quantities obey the inviscid compressible Euler equations, that is, the first three moments of Vlasov's equation (see [11] for details), which read:

$$
\begin{gathered}
\frac{\partial n_{i}}{\partial t}+\frac{\partial j_{i}}{\partial x}=0 \\
\frac{\partial j_{i}}{\partial t}+\frac{\partial}{\partial x}\left[\frac{j_{i}^{2}}{n_{i}}+p_{i}\right]=\mu_{i} n_{i} E \\
\frac{\partial w_{i}}{\partial t}+\frac{\partial}{\partial x}\left[\left(w_{i}+p_{i}\right) \frac{j_{i}}{n_{i}}+K_{i}\right]=\mu_{i} j_{i} E .
\end{gathered}
$$

In the absence of collisions, there is no mechanism to ensure the relaxation of the distribution functions towards Maxwellian equilibrium. So, we have to assume some equation of state between the fluid quantities in order to close the Euler system (7-9).

In this article, the use of the fluid approximation will be justified based on the following physical hypotheses. First, we assume that the thermal velocities of the two species are several orders of magnitude apart. Then, we suppose that the characteristic speed of the phenomenon under consideration is small with respect to the thermal velocity of the electrons, but large when compared to that of the ions.

As a consequence, the two species react quite distinctly to a perturbation. During the time scale of the perturbation, the electrons have more than enough time to thermalise, hence their temperature $\theta_{1}$ will remain constant in space and time, and equal to its initial value $\theta_{1}^{0}$. On the contrary, the ions will not have time to exchange any heat with their neighbours, so $\partial_{x} K_{2}$ will be 
negligible with respect to $\partial_{t} w_{2}$. Summarising, the electrons will be described by the isothermal two-moment system

$$
\begin{aligned}
\frac{\partial n_{1}}{\partial t}+\frac{\partial j_{1}}{\partial x} & =0 \\
\frac{\partial j_{1}}{\partial t}+\frac{\partial}{\partial x}\left[\frac{j_{1}^{2}}{n_{1}}+p_{1}\right] & =-\frac{e}{m_{1}} n_{1} E \\
\text { where: } \quad p_{1} & =\theta_{1}^{0} n_{1}
\end{aligned}
$$

while the ions will obey the adiabatic three-moment system

$$
\begin{aligned}
\frac{\partial n_{2}}{\partial t}+\frac{\partial j_{2}}{\partial x} & =0 \\
\frac{\partial j_{2}}{\partial t}+\frac{\partial}{\partial x}\left[\frac{j_{2}^{2}}{n_{2}}+p_{2}\right] & =\frac{Z e}{m_{2}} n_{2} E \\
\frac{\partial w_{2}}{\partial t}+\frac{\partial}{\partial x}\left[\left(w_{2}+p_{2}\right) \frac{j_{2}}{n_{2}}\right] & =\frac{Z e}{m_{2}} j_{2} E \\
\text { where: } p_{2} & =2 w_{2}-\frac{j_{2}^{2}}{n_{2}} .
\end{aligned}
$$

The motion of both species will still be coupled through Poisson's equation

$$
-\frac{\partial^{2} \phi}{\partial x^{2}}=\frac{\partial E}{\partial x}=\frac{e}{\varepsilon_{0}}\left(Z n_{2}-n_{1}\right) .
$$

The periodicity conditions

$$
\left\{n_{1}, j_{1}, n_{2}, j_{2}, w_{2}, E\right\}(t, x+L)=\left\{n_{1}, j_{1}, n_{2}, j_{2}, w_{2}, E\right\}(t, x)
$$

as well as the initial conditions

$$
n_{i}(0, x)=n_{i}^{0}(x), \quad j_{i}(0, x)=n_{i}^{0}(x) u_{i}^{0}(x), \quad w_{2}(0, x)=\frac{n_{2}^{0}(x)}{2}\left[u_{2}^{0}(x)^{2}+\frac{k_{\mathrm{B}} T_{2}^{0}}{m_{2}}\right],
$$

are inherited from the kinetic description (3-4) and the pressure law (16). The set of equations (10-19) will be referred to as the two-species Euler-Poisson model.

Remark: A simple, but somewhat lengthy, calculation on the adiabatic threemoment system (13-16) shows that

$$
\frac{1}{p_{2}} \frac{\mathrm{d} p_{2}}{\mathrm{~d} t}-\frac{3}{n_{2}} \frac{\mathrm{d} n_{2}}{\mathrm{~d} t}=0, \quad \text { where: } \quad \frac{\mathrm{d}}{\mathrm{d} t}=\frac{\partial}{\partial t}+u_{2} \frac{\partial}{\partial x},
$$


is the material (or convective) derivative along the ion trajectories. This is integrated as

$$
p_{2}=A n_{2}^{3}
$$

which is often referred to as "the adiabatic law", or "the polytropic law". However, one should realise that the "constant" $A$ depends a priori on the trajectory and that, in the absence of collisions, no mechanism can uniformise its values. This is why - following the usual computational approach [12, pp. 399 sqq.] to inviscid compressible gas dynamics - we keep a three-moment description of the ions, instead of closing the fluid hierarchy at the level of $j_{2}$ and using the pressure law (20).

In fact, a two-moment, two-species system has been studied previously in [1316]. In these works, ions and electrons are considered as isothermal species (although some of the results can be generalised for the polytropic law). As pointed out above, in a collisionless plasma there is no reason either to consider the ions as isothermal nor to reduce the three-moment description to the twomoment one. This will also be checked numerically in the test case of ion acoustic waves.

\subsection{Rescaled equations}

Given some units of length, speed, and density: $\bar{x}, \bar{v}, \bar{n}$, the natural units of time, current, energy density, pressure, and distribution function are set to:

$$
\bar{t}=\bar{x} / \bar{v}, \quad \bar{\jmath}=\bar{n} \bar{v}, \quad \bar{w}=\bar{p}=\bar{n} \bar{v}^{2}, \quad \bar{f}=\bar{n} / \bar{v} .
$$

Then, the choice of some unit $\bar{\mu}$ for the ratio $\mu$ yields, from the equipartition of energy theorem, the natural unit of potential and hence that of electric field:

$$
\bar{\phi}=\bar{v}^{2} / \bar{\mu}, \quad \bar{E}=\bar{v}^{2} /(\bar{\mu} \bar{x}) .
$$

As the electron motions are the most rapid, they will set the pace of the codes, either kinetic or fluid. Moreover, in a hot plasma, their fluid velocity will always be small compared to their thermal velocity. Hence, it looks sensible, from a computational point of view, to set

$$
\bar{\mu}=\left|\mu_{1}\right|=\frac{e}{m_{1}} \quad \text { and } \quad \bar{v}=v_{\mathrm{th} 1}=\sqrt{\frac{k_{\mathrm{B}} T_{1}}{m_{1}}} .
$$


There remains to choose $\bar{x}$ and $\bar{n}$. The periodic character of the system suggests to set

$$
\bar{x}=L \quad \text { and } \quad \bar{n}=\frac{1}{L} \int_{0}^{L} n_{1}^{0}(x) \mathrm{d} x .
$$

Another possible scaling - relevant in some circumstances - is to set $\bar{x}=\lambda_{\mathrm{D} 1}$, the electron Debye length. As far as our problem is concerned, it has several drawbacks: mathematically, it typically makes the domain of study look very large; physically, it somewhat obscures the quasi-neutral limit; numerically, it is less related to the computational cost.

\subsubsection{Rescaled Vlasov-Poisson model}

Keeping the same notations $t, x, v$ and $f, E$ for the rescaled unknowns and variables, the equations (1-4) become:

$$
\begin{aligned}
& \frac{\partial f_{1}}{\partial t}+v \frac{\partial f_{1}}{\partial x}-E \frac{\partial f_{1}}{\partial v}=0 \\
& \frac{\partial f_{2}}{\partial t}+v \frac{\partial f_{2}}{\partial x}+\gamma E \frac{\partial f_{2}}{\partial v}=0 \\
& -\eta^{2} \frac{\partial^{2} \phi}{\partial x^{2}}=\eta^{2} \frac{\partial E}{\partial x}=\int_{-\infty}^{+\infty}\left(Z f_{2}-f_{1}\right) \mathrm{d} v \\
& f_{i}(t, x+1, v)=f_{i}(t, x, v), i=1,2, \quad E(t, x+1)=E(t, x) \\
& f_{1}(0, x, v)=n_{1}^{0}(x) \mathcal{M}_{1}\left(v-u_{1}^{0}(x)\right), \quad f_{2}(0, x, v)=\frac{1}{Z} n_{2}^{0}(x) \mathcal{M}_{\alpha}\left(v-u_{2}^{0}(x)\right)
\end{aligned}
$$

The dimensionless parameters $\gamma, \alpha, \eta$ are defined as

$$
\gamma=\left|\frac{\mu_{2}}{\mu_{1}}\right|=\frac{Z m_{1}}{m_{2}}, \quad \alpha=\frac{\theta_{2}^{0}}{\theta_{1}^{0}}=\frac{m_{1} T_{2}^{0}}{m_{2} T_{1}^{0}}, \quad \eta=\frac{\bar{v}}{\bar{x}} \sqrt{\frac{m_{1} \varepsilon_{0}}{e^{2} \bar{n}}}=\frac{\lambda_{\mathrm{D} 1}}{L}=\frac{1}{\bar{t} \omega_{\mathrm{p} 1}}
$$

where $\omega_{\mathrm{p} 1}$ is the electron plasma pulsation. It will prove convenient to introduce the parameter $\beta$ satisfying

$$
\alpha=\beta \gamma, \quad \text { i.e. } \quad \beta=\frac{T_{2}^{0}}{Z T_{1}^{0}} .
$$

Physically, the parameter $\gamma$ is known to be quite small: as the protons contribute to the charge of the ion, but not the neutrons, one has: $\gamma \leq m_{1} / m_{\text {proton }} \simeq$ 
$1 / 1836$. As for $\beta$, it can be arbitrary: in laser-plasma interaction in the subpicosecond regime, the electron temperature may reach very high values as compared to the ion temperature and the parameter $\beta$ can be very small; however, the collisions that are inevitable in a real plasma tend to equalise the temperatures, so we can expect $\beta$ to be never very large. Therefore, we can expect $\alpha$ to be small, too.

Finally, the numerical simulation of the plasma by the Vlasov-Poisson model demands that

(1) The collective description of the plasma be physically valid on the length scale of the simulation box. For evident computational reasons, the latter is taken as one space period, hence, $\eta$ must be small enough.

(2) The effects of collisions can be neglected. A necessary (but not sufficient on long time scales) condition for this is that the graininess parameter $\mathrm{g} \ll 1$, where:

$$
\mathrm{g} \stackrel{\text { def }}{=}\left[\bar{n} \lambda_{\mathrm{D} 1}^{3}\right]^{-1}=\left(\frac{\varepsilon_{0} k_{\mathrm{B}}}{e^{2}}\right)^{-3 / 2} \bar{n}^{1 / 2} T_{1}^{-3 / 2} .
$$

\subsubsection{Rescaled Euler-Poisson model}

After straightforward calculations, we get:

$$
\begin{aligned}
& \frac{\partial n_{1}}{\partial t}+\frac{\partial j_{1}}{\partial x}=0 \\
& \frac{\partial j_{1}}{\partial t}+\frac{\partial}{\partial x}\left[\frac{j_{1}^{2}}{n_{1}}+n_{1}\right]=-n_{1} E \\
& \text { i.e.: } p_{1}=n_{1} \\
& \frac{\partial n_{2}}{\partial t}+\frac{\partial j_{2}}{\partial x}=0 \\
& \frac{\partial j_{2}}{\partial t}+\frac{\partial}{\partial x}\left[\frac{j_{2}^{2}}{n_{2}}+p_{2}\right]=\gamma n_{2} E \\
& \frac{\partial w_{2}}{\partial t}+\frac{\partial}{\partial x}\left[\left(w_{2}+p_{2}\right) \frac{j_{2}}{n_{2}}\right]=\gamma j_{2} E \\
& \text { where: } p_{2}=2 w_{2}-\frac{j_{2}^{2}}{n_{2}} . \\
&-\eta^{2} \frac{\partial^{2} \phi}{\partial x^{2}}=\eta^{2} \frac{\partial E}{\partial x}=Z n_{2}-n_{1}, \\
&\left\{n_{1}, j_{1}, n_{2}, j_{2}, w_{2}, E\right\}(t, x+1)=\left\{n_{1}, j_{1}, n_{2}, j_{2}, w_{2}, E\right\}(t, x)
\end{aligned}
$$




$$
n_{i}(0, x)=n_{i}^{0}(x), \quad j_{i}(0, x)=n_{i}^{0}(x) u_{i}^{0}(x), \quad w_{2}(0, x)=\frac{n_{2}^{0}(x)}{2}\left[u_{2}^{0}(x)^{2}+\alpha\right]
$$

\section{The limit regimes}

We now examine the asymptotic behaviour of the Vlasov-Poisson and EulerPoisson models when the various parameters tend to zero. These derivations are purely formal and we do not investigate the combined effects of several parameters tending to zero as functions of one another.

\subsection{The cold ion regime}

If the parameter $\alpha$ is zero, the ions are initially monokinetic, or "cold" (in the kinetic language), or pressureless (in the hydrodynamic language). It is not difficult to prove that they remain so with time, namely:

- the solution to $(22)$ is $f_{2}(t, x, v)=n_{\mathrm{ci}}(t, x) \delta\left(v-\frac{j_{\mathrm{ci}}(t, x)}{n_{\mathrm{ci}}(t, x)}\right)$;

- the solution to $(32-35)$ is: $\left\{n_{2}, j_{2}, w_{2}\right\}(t, x)=\left\{n_{\mathrm{ci}}, j_{\mathrm{ci}}, \frac{j_{\mathrm{ci}}^{2}}{2 n_{\mathrm{ci}}}\right\}(t, x)$,

where $\left(n_{\mathrm{ci}}, j_{\mathrm{ci}}\right)$ solves the pressureless Euler system

$$
\frac{\partial n_{\mathrm{ci}}}{\partial t}+\frac{\partial j_{\mathrm{ci}}}{\partial x}=0, \quad \frac{\partial j_{\mathrm{ci}}}{\partial t}+\frac{\partial}{\partial x} \frac{j_{\mathrm{ci}}^{2}}{n_{\mathrm{ci}}}=\gamma n_{\mathrm{ci}} E, \quad\left\{n_{\mathrm{ci}}, j_{\mathrm{ci}}\right\}(0, x)=n_{2}^{0}(x)\left\{1, u_{2}^{0}(x)\right\} .
$$

\subsection{The one-species regime}

If $\gamma$ is negligible, the ions are infinitely massive, so that they do not react any more to the electric force. The third term vanishes in (22), and it is easy to check that this equation, with the initial condition (25), admits the solution

$$
f_{2}(t, x, v)=n_{2}^{0}(x-v t) \mathcal{M}_{\alpha}\left(v-u_{2}^{0}(x-v t)\right) .
$$

Similarly, the right-hand sides in (33) and (34) vanish; however, the solution to this simplified problem generally cannot be written in closed form. As for the electrons, their distribution function is solution to (21) and (23), where $f_{2}$ is now a data given by the formula (40). Similarly, their density and current are solution to (29-30) coupled with (36), with $n_{2}$ given as the solution of a decoupled problem. We will refer to this situation as the one-species regime. 
In the kinetic formulation, $n_{2}$ is given by

$$
n_{2}(t, x)=\int_{-\infty}^{+\infty} f_{2}(t, x, v) \mathrm{d} v=\int_{-\infty}^{+\infty} n_{2}^{0}(x-\xi) \mathcal{M}_{\alpha}\left(\frac{\xi-t u_{2}^{0}(x-\xi)}{t}\right) \frac{\mathrm{d} \xi}{t}
$$

this allows to calculate it explicitly in particular cases. For instance, supposing that $u_{2}^{0}(x)=U=$ const, we use the identity $\mathcal{M}_{\alpha}(\xi / t) / t=\mathcal{M}_{\alpha t^{2}}(\xi)$ and we find

$$
n_{2}(t, x)=\left(n_{2}^{0} * \tau_{t U} \mathcal{M}_{\alpha t^{2}}\right)(x)=\left(\tau_{t U} n_{2}^{0} * \mathcal{M}_{\alpha t^{2}}\right)(x),
$$

where $*$ and $\tau$. denote the convolution and translation operators, respectively. In other words, the ion density undergoes an advection at the speed $U$, while the diffusion of rapid particles simultaneously blurs out the discrepancy between dense and less dense regions.Let us finally remark that the density $n_{2}$ satisfies the linear diffusion equation:

$$
\frac{\partial n_{2}}{\partial t}+U \frac{\partial n_{2}}{\partial x}=\frac{\alpha}{2} t \frac{\partial^{2} n_{2}}{\partial x^{2}}
$$

as it can be easily checked by a direct computation. As more particular cases:

- if the ion density is initially constant $\left(n_{2}^{0}(x) \equiv Z^{-1}\right)$, it will remain so with time,

- if the ions are initially monokinetic $(\alpha=0)$, they will remain so, and the density profile $n_{2}^{0}$ will simply propagate at the speed $U$.

\subsection{Quasi-neutrality}

When $\eta \rightarrow 0$, the equation (23) or (36) show that the plasma tends to become neutral, i.e.

$$
n_{1}=Z n_{2}
$$

This equation, however, is often too crude to derive any interesting physics. A subtler argument is that the plasma pulsations $\omega_{\mathrm{p} 1} \propto \eta^{-1}$ and $\omega_{\mathrm{p} 2} \propto \gamma^{1 / 2} \eta^{-1}$ become infinite (provided $\gamma$ remains finite). The particles follow instantaneously the electric field, so that their distribution function is at any time in an equilibrium state described by the Boltzmann factor, hence [17, p. 14]:

$$
n_{i} \propto \mathrm{e}^{-\mu_{i} \phi / \theta_{i}}
$$


where $\phi$ is the electric potential.

A quasineutral limit $(\eta \rightarrow 0)$ at the level of the fluid description has been obtained in $[16,14]$ for a two-species isothermal (or polytropic) 2-moment system. On the contrary, the derivation of an asymptotic dynamic in the kinetic description seems difficult. It is possible to derive formally a hydrodynamic limit (generalising [18] to the two-species case) in the case of cold ions and electrons. However, a vanishing electron temperature cannot represent the physics of totally ionised plasmas - which, by the way, is one reason for choosing $\bar{v}=v_{\text {th1 }}$. So, we shall adopt (42) or (43) as operational definitions of quasi-neutrality. Because of the disparity between the two plasma pulsations, it can happen that electrons be "more quasi-neutral" than ions, i.e. (43) is better satisfied for $i=1$ than $i=2$.

This is even necessary to avoid some incoherences. Indeed, we shall see that assuming (43) for both species is incompatible with the physical hypotheses of periodicity and global neutrality.

For the sake of simplicity, let us assume that we are not dealing with too weak solutions, i.e. that the densities $n_{i}$ are integrable functions on $(0,1)$, hence locally integrable on $\mathbb{R}$. In the kinetic framework, this is the case e.g. if the distribution functions $f_{i}$ are initially integrable functions on $(0,1)_{x} \times \mathbb{R}_{v}$ : then they will remain so with time, and $n_{i}$ will be integrable on $(0,1)$. Thus, by (23) or (36), $E$ will be continuous, and $\phi$ continuously differentiable.

As the potential is at any time solution to a static equation, we forget the $t$-dependence in the rest of this Section and we denote by ' the $x$-derivative. Moreover, we set the additive constant of the potential by imposing $\phi(0)=0$. Then, Eq. (36) with the hypothesis (43) reads:

$$
-\eta^{2} \phi^{\prime \prime}(x)=Z n_{2}(0) \mathrm{e}^{-\gamma \phi(x) / \theta_{2}(x)}-n_{1}(0) \mathrm{e}^{\phi(x) / \theta_{1}(x)}
$$

Let us consider the linear regime with the following assumptions:

- $\phi \ll \theta_{i}, i=1,2$, i.e., in physical units, the value of the potential energy of a particle is small as compared to the value of the temperature in energy units;

- the temperatures $\theta_{i}$ are nearly uniform and equal to their characteristic values $\bar{\theta}_{1}=1$ and $\bar{\theta}_{2}=\alpha$.

Then (44) becomes:

$$
-\eta^{2} \phi^{\prime \prime}(x)+k^{2} \phi(x)=Z n_{2}(0)-n_{1}(0)=a .
$$


where: $k^{2}=Z n_{2}(0) / \beta+n_{1}(0)$. Finally, $\phi$ is characterised by the conditions $\phi$ satisfies (45) for all $x \in \mathbb{R}, \quad \phi(0)=0$, $\phi$ is continuously differentiable and has the period 1 ,

which are clearly equivalent to

$$
\phi \text { satisfies }(45) \text { for all } x \in(0,1), \quad \phi(0)=\phi(1)=0, \quad \phi^{\prime}(0)=\phi^{\prime}(1) \text {. }
$$

Now, the solution to (45) with the boundary conditions $\phi(0)=\phi(1)=0$ is

$$
\phi(x)=\frac{a}{k^{2}} \times \frac{1-\mathrm{e}^{k x / \eta}+\mathrm{e}^{k / \eta}\left(1-\mathrm{e}^{-k x / \eta}\right)}{1+\mathrm{e}^{k / \eta}},
$$

which satisfies:

$$
\phi^{\prime}(0)=\frac{a}{k \eta} \times \frac{\mathrm{e}^{k / \eta}-1}{1+\mathrm{e}^{k / \eta}}=-\phi^{\prime}(1) .
$$

In other words, there is no periodic function satisfying (45) on the whole of $\mathbb{R}$, unless $a=0$, in which case nothing happens: the potential and the densities are uniform and constant in time. Equivalently, it is not difficult to check that the densities $n_{1}=(1+\phi) n_{1}(0)$ and $n_{2}=(1-\phi / \beta) n_{2}(0)$, with $\phi$ given by (46), do not agree with the global neutrality condition on $(0,1)$ unless $a=0$.

So far, we have not disproved the existence of a non-trivial periodic solution to (44), i.e. the possibility that (43) be satisfied for both species in the nonlinear regime. But if we start from a neutral plasma $\left(n_{1}=n_{2}\right.$ everywhere) with uniform temperatures $\theta_{1}$ and $\theta_{2}$, it should go through a phase of linear behaviour for the potential like above. During this phase, the condition (43) must break down for at least one species. According to the plasma pulsation argument, this should happen first for the ions.

The case where (43) holds for the electrons only is investigated in Appendix A. Unlike the previous situation, it appears coherent with the physical hypotheses. And, at least in the linear potential regime and in the kinetic framework, it satisfies

$$
Z n_{2}-n_{1} \propto \eta^{2}
$$

which is precisely what (36) says, provided $\phi^{\prime \prime}(x)$ remains finite. This confirms that the scaling of Section 2.3 is the relevant one for dealing with the quasineutrality issue. 


\section{The model case: Ion acoustic waves}

As explained in the Introduction, it is not necessary to use the full VlasovMaxwell system and a complicated geometry for testing the validity of the physical assumptions used in the modelling of real-life problems. Certain instances of the one-dimensional Vlasov-Poisson system are good candidates for testing the more general hypotheses made e.g. in the study of turbulence in tokamak plasmas, while being far simpler both from a mathematical and a computational point of view. There is no need, for instance, to cope with the burden of Larmor radius effects, and so on.

As an example of such a physical phenomenon, satisfying the hypotheses of Section 2.2, we selected the ion acoustic waves [19]. We assume that electrons and ions are described by the systems (10-12) and (13-16) respectively, and that (43) holds for $i=1$.

Under these circumstances, one shows (see [17, p. 262]) that small perturbations of the ion density admit the dispersion relation

$$
\frac{\omega^{2}}{\mathrm{k}^{2}}=\frac{k_{\mathrm{B}}}{m_{2}}\left(\frac{Z T_{1}}{1+\mathrm{k}^{2} \lambda_{\mathrm{D} 1}^{2}}+3 T_{2}\right) .
$$

As noted above, we simulate only one wavelength, thus choosing $\mathrm{k}=2 \pi / L$. As $\eta$ must be small, so is $\mathrm{k} \lambda_{\mathrm{D} 1}=2 \pi \eta$. In this case, the waves have a constant "sound" speed

$$
c_{\mathrm{s}}=\sqrt{k_{\mathrm{B}}\left(Z T_{1}+3 T_{2}\right) / m_{2}} .
$$

This result is also obtained by using the quasi-neutrality condition (42); so one checks that taking $\eta \rightarrow 0$ does correspond to making the charge balance more exact. Comparing $c_{\mathrm{s}}$ to the thermal velocities of both species, we find:

$$
\begin{aligned}
& c_{\mathrm{s}} / v_{\mathrm{th} 1}=\sqrt{\gamma(1+3 \beta)}, \\
& c_{\mathrm{s}} / v_{\mathrm{th} 2}=\sqrt{\beta^{-1}(1+3 \beta)} .
\end{aligned}
$$

Thus, it is sufficient to suppose both $\beta$ and $\gamma$ quite small in order to satisfy $a$ posteriori the assumptions of the hydrodynamic derivation.

In order to undertake a kinetic analysis of these waves based on the two-species Vlasov-Poisson system, we must take care that the characteristic time of the phenomenon be small as compared to the collision time. From [17, pp. 172-3], 
we get the expressions of electron-electron, electron-ion, and ion-ion collision frequencies, which we rewrite as functions of our dimensionless parameters and of the electron plasma pulsation $\omega_{\mathrm{p} 1}$ :

$$
\begin{aligned}
\nu_{11}=\nu_{12} / Z & =\left(\sqrt{2} \ln \Lambda / 12 \pi^{3 / 2}\right) \mathrm{g} \omega_{\mathrm{p} 1} ; \\
\nu_{22} & =\left(\ln \Lambda / 12 \pi^{3 / 2}\right) Z \mathrm{~g} \gamma^{1 / 2} \beta^{-3 / 2} \omega_{\mathrm{p} 1} .
\end{aligned}
$$

The values of the Coulomb logarithm $\ln \Lambda$ vary little within the range of natural and laboratory plasmas [17, p. 170]; we shall take $\ln \Lambda=18$ as a typical (and rather overestimated in our test-cases) value. Assuming that the formula (48) is still approximately valid, we obtain an estimation of the average number of electron-electron and ion-ion collisions occurring during one period $\mathrm{T}=L / c_{\mathrm{s}}$ of the sound wave:

$$
\begin{aligned}
& C_{11}=\nu_{11} \mathrm{\top}=0.38 \mathrm{~g} \frac{L \omega_{\mathrm{p} 1}}{v_{\mathrm{th} 1}} \frac{v_{\text {th } 1}}{c_{\mathrm{s}}}=0.38 \mathrm{~g} \eta^{-1} \gamma^{-1 / 2}(1+3 \beta)^{-1 / 2}, \\
& C_{22}=\nu_{22} \mathrm{\top}=0.27 \mathrm{~g} \eta^{-1} \beta^{-3 / 2}(1+3 \beta)^{-1 / 2} .
\end{aligned}
$$

So, the Vlasov-Poisson analysis of the ion acoustic waves is physically justified provided $C_{12}=Z C_{11}$ and $C_{22}$ are small. The kinetic treatment [17, p. 425] confirms qualitatively the fluid analysis, yet finds a Landau damping rate

$$
r=\sqrt{\pi / 8} \mathrm{k} c_{\mathrm{s}}\left[\gamma^{1 / 2}+\beta^{-3 / 2} \mathrm{e}^{-1 / 2 \beta}\right]
$$

The damping factor over one period is

$$
G \equiv \mathrm{e}^{-r \mathrm{\top}}=\exp \left[-\sqrt{\frac{\pi^{3}}{2}}\left(\gamma^{1 / 2}+\beta^{-3 / 2} \mathrm{e}^{-1 / 2 \beta}\right)\right]
$$

Though this estimation is rigorously valid only in the $\beta \ll 1$ (and $\eta \ll 1$ ) limit, it can serve as a "confidence rate" for the fluid modelling: the lower the $G$, the less appropriate the fluid treatment certainly is. Table 1 gives diverse values of $G$.

The values $\gamma^{-1}=1836,3670$ and 5496 correspond resp. to ordinary hydrogen, deuterium (and also, approximately, helium 4), and tritium. The value $\beta^{-1}=$ 23 is the least integer $b$ such that $\exp \left[-\sqrt{\pi^{3} / 2} b^{3 / 2} \mathrm{e}^{-b / 2}\right]>0.995$. 
Table 1

\begin{tabular}{|c|c|c|c|c|c|c|}
\hline$\beta^{-1} \backslash \gamma^{-1}$ & 100 & 900 & 1836 & 3670 & 5496 & $\infty$ \\
\hline 2 & 0.011 & 0.015 & 0.015 & 0.016 & 0.016 & 0.017 \\
\hline 5 & 0.018 & 0.024 & 0.025 & 0.025 & 0.026 & 0.027 \\
\hline 10 & 0.29 & 0.38 & 0.39 & 0.40 & 0.41 & 0.43 \\
\hline 15 & 0.59 & 0.77 & 0.80 & 0.83 & 0.84 & 0.88 \\
\hline$\geq 23$ & 0.67 & 0.88 & 0.91 & 0.94 & 0.95 & 1. \\
\hline
\end{tabular}

"Confidence rate" for the fluid treatment of ion acoustic waves

\section{Numerical implementation}

\subsection{The method}

Our choice of a numerical method was guided by the following requirements. First, the method should be accurate enough to make meaningful comparisons between the two-species Vlasov-Poisson system and its fluid, one-species and cold-ion approximations, as well as meaningful measurements on the quasineutrality criteria. Thus, we rejected the too noisy PIC methods for the kinetic simulations, and we were led to prefer a true "Vlasovian" method, i.e. a numerical scheme designed to solve the Vlasov equation as a partial differential equation posed in phase space. (See e.g. [20] for a review.) Then, we desired a method which could also be applied to the fluid equations, in order to lessen the impact of method-dependent errors on the comparisons.

This led us to choose the Weighted Essentially Non-Oscillatory (WENO) flux reconstruction methods [12] (see also [21-23]). They can be applied to any hyperbolic system of conservation laws; yet both the Vlasov and the Euler equations belong in this framework. They have the double advantage of being able to deal with shocks (which typically appear in fluid equations) or steep gradients (which typically appear in Vlasov's equation, cf. Figures 10 and 12 below), while achieving high-order accuracy in the smooth regions. This makes them among the most precise methods available for conservation laws.

As the main drawback, they do not guarantee the conservation of any physical invariant other than the one furnished by the conservation law itself. This is of no importance for the Euler-Poisson model, which expresses the conservation of all physically interesting invariants: mass, momentum and energy. On the other hand, a WENO Vlasov-Poisson code cannot enforce the conserva- 
tion of any moment of $f_{i}$ above the order 0 , and especially that of the total energy $W(t)$.

Neither do these methods preserve the positivity of the distribution function, or of any positive function. This, however, is no big hindrance since the negative values that may appear are usually very small in absolute value. From a physical point of view, this problem may affect only some phase space regions of high momentum, where the density is so small that there are no particles to interact with existing high phase velocity waves.

We chose a fifth-order, flux-splitting, WENO scheme, associated to a thirdorder total variation diminishing (TVD) Runge-Kutta method for the time discretisation. The implementation of the two-species Vlasov-Poisson system (1-4) poses no difficulty, thanks to the one-dimensional character of the unknown $f_{i}$, and the to easy determination of the wind direction. The code was based on a Boltzmann-Poisson code already used in [24,25].

The implementation of the two-species Euler-Poisson model (10-19) is much more complex, because of its non-linearity and of the dimension 2 or 3 . In order to achieve the desirable accuracy, it is preferable to perform local base changes (this is known as "characteristic WENO"), and Lax-Friedrichs flux splitting before applying WENO reconstruction, and then to go back to the physical fluxes [12, p. 368]. For the expressions of the base changes cf. [26]. However, this is not possible for the pressureless Euler system (39): this system is no longer hyperbolic, so there is no characteristic base. Thus we had to do non-characteristic WENO with flux splitting in this case.

Finally, one-species simulations based on the same principles have also been implemented, both in the kinetic and fluid approaches.

\subsection{Cost v. efficiency analysis}

As usual, the coexistence of multiple scales has a high computational cost. Indeed, the time step in an explicit Vlasov code is given by the formula

$$
\Delta t=g\left[R v_{\text {th } 1} \frac{N_{x}}{L}+\frac{e}{m_{1}} E_{\text {phys }}^{\infty}(t) \frac{N_{v}}{R v_{\text {th } 1}}\right]^{-1},
$$

where: $E_{\text {phys }}^{\infty}(t)$ is the supremum of the modulus of the electric field (in physical units) at time $t ; N_{x}$ and $N_{v}$ are the respective numbers of mesh points in $x$ and $v ; R$ is a constant which controls the extension of the $v$-space, and has to 
be taken large enough to prevent the numerical loss of particles at its ends; and $g<1$ is used to ensure the CFL condition. Hence

$$
\Delta t=g\left[R N_{x}+E_{\mathrm{resc}}^{\infty}(t) \frac{N_{v}}{R}\right]^{-1} \bar{t} \simeq \frac{\bar{t}}{C N},
$$

where $E_{\text {resc }}^{\infty}(t)$ is now the supremum of the electric field in rescaled units, $N=\max \left(N_{x}, N_{v}\right)$, and $C$ is a somewhat large dimensionless constant.

On the other hand, the computation time necessary for advancing one time step is approximately $a_{\text {kin }} N_{x} N_{v}$, for some constant $a_{\text {kin }}$. So, the order of magnitude of the time taken by the kinetic code to compute one period is

$$
t_{\text {kin }}(\mathrm{T})=a_{\text {kin }} N_{x} N_{v} \frac{\mathrm{T}}{\Delta t}=a_{\text {kin }} N_{x} N_{v} \frac{C N}{\bar{t}} \frac{L}{c_{\mathrm{s}}} \simeq \frac{a_{\text {kin }} C N^{3}}{\sqrt{\gamma}} .
$$

Moreover, large values of $N(N \simeq 200)$ are necessary to obtain a good numerical conservation of energy, which is mandatory for testing the thermodynamic hypotheses of the fluid derivation (see Section 6.5 below), since more than $99 \%$ of the total energy of the system is in the form of thermal agitation, i.e. internal energy. This makes $t_{\text {kin }}(T)$ quite large even for $\gamma=1 / 100$, i.e. well above its physical range of values.

Of course a Euler code does much better, because is considers only 6 unknowns for each point in the $x$-mesh, instead of $2 N_{v}+1 \gg 1$. For isothermal electrons, the formula for the time step is

$$
\Delta t=\frac{g L}{N_{x}}\left(v_{\mathrm{th} 1}+u_{\mathrm{phys}}^{\infty}(t)\right)^{-1} \simeq \frac{g}{N_{x}} \bar{t}
$$

since $u_{\mathrm{phys}}^{\infty}(t)$, the supremum of the modulus of the electron fluid velocity (in physical units) at time $t$, is always negligible before $v_{\text {th1 }}$. The computation time for advancing one time step is now $\simeq a_{\mathrm{flu}} N_{x}$, for some constant $a_{\mathrm{flu}}$. So, we find that the time taken by the fluid code to compute one period is roughly equal to

$$
t_{\mathrm{flu}}(\mathrm{T})=a_{\mathrm{flu}} N_{x} \frac{\mathrm{T}}{\Delta t} \simeq \frac{a_{\mathrm{flu}} N^{2}}{g \sqrt{\gamma}} .
$$

We still have the unpleasant dependence in $\gamma^{-1 / 2}$, but the numerator is now in $N^{2}$. Typically, $t_{\text {kin }}(\mathrm{T}) / t_{\text {flu }}(\mathrm{T}) \simeq 10^{3}$. This makes the fluid simulation much more affordable for very small values of $\gamma$, which precisely should make it more accurate. 


\section{$6 \quad$ Numerical tests}

\subsection{Test cases}

The instances of the Vlasov-Poisson and Euler-Poisson problems are specified with the initial values of density, fluid velocity, and temperature $n_{i}^{0}(x), u_{i}^{0}(x), T_{i}^{0}$; the length of the simulation box $L$ (which is equal to wavelength of the simulated wave); and the mass $m_{2}$ and charge number $Z$ of the ions. We tested the following values:

- The electrons had an initial temperature $T_{1}^{0}=100 \mathrm{eV}$ and an average density $\bar{n}=10^{16} \mathrm{~m}^{-3}$. Hence their thermal velocity was $v_{\text {th1 }}=4.19 \times$ $10^{6} \mathrm{~m} / \mathrm{s}$, and the Debye length was $\lambda_{\mathrm{D} 1}=743 \mu \mathrm{m}$. Moreover, the graininess parameter was $g=2.44 \times 10^{-7}$. The modelling by the Vlasov-Poisson system is thus excellent on short time scales.

- The species 2 was a "light proton", with charge $+e$, whose mass was taken as 100 or 900 times that of the electron. Indeed, kinetic computations with $\gamma=1 / 900$ were already very heavy, while Table 1 shows that this value does not deteriorate much the confidence rate with respect to the physical values for the three hydrogen ions.

The initial temperature of the light protons was taken either as $T_{2}^{0}=1$ or $10 \mathrm{eV}(\beta=1 / 10$ or $1 / 100)$.

- The length of the simulation box was $L=1$ or $5 \mathrm{~cm}$, corresponding to time scales $\bar{t}=2.38$ or $11.9 \mathrm{~ns}$, and to $\eta=7.43 \times 10^{-2}$ or $1.49 \times 10^{-2}$.

Hence, the values of the collision-related parameters $C_{12}=C_{11}$ and $C_{22}$ defined by (51-52) were bounded in the worst cases as $C_{12} \leq 1.9 \times 10^{-4}$ and $C_{22} \leq 4.4 \times 10^{-3}$. This fully justifies the modelling of the plasma by the Vlasov-Poisson system over a few periods of the ion acoustic waves.

- Both species were initially at rest: $u_{1}^{0}(x) \equiv u_{2}^{0}(x) \equiv 0$.

- The initial density profiles were $n_{1}^{0}(x)=n_{2}^{0}(x)=\bar{n} \psi(2 \pi x / L)$ where: $\psi(\vartheta) \equiv 1+\varepsilon \cos \vartheta \exp \sin \vartheta$. The value of the parameter $\varepsilon$ was 0.05 .

- One-species simulations were performed with the same initial conditions $n_{1}^{0}, u_{1}^{0}, T_{1}^{0}$ for the electrons as above. In order to mimic the two-species case, the initial density of the neutralising background was $n_{2}^{0} \equiv n_{1}^{0}$. Then, this density was taken as

$$
n_{2}(t, x)=\bar{n}\left(1+\varepsilon \cos \frac{2 \pi x}{L} \exp \sin \frac{2 \pi x}{L} \exp \left[-\frac{2 \pi^{2}}{L^{2}} \frac{k_{\mathrm{B}} T_{1}^{0}}{m_{1}} \alpha t^{2}\right]\right)
$$

which is a fairly good approximation of the exact solution (41) for $U=0$.

These different test-cases were numbered as shown in Table 2. This numbering will be used in all subsequent figures. 
Table 2

\begin{tabular}{|c|c|c|c|c|}
\hline$\eta$ & \multicolumn{2}{|c|}{$7.43 \times 10^{-2}$} & \multicolumn{2}{|c|}{$1.49 \times 10^{-2}$} \\
\hline$\beta^{-1} \backslash \gamma^{-1}$ & 100 & 900 & 100 & 900 \\
\hline 10 & 1 & 3 & 6 & 8 \\
\hline 100 & 4 & 2 & 5 & 7 \\
\hline
\end{tabular}

Values of $\beta, \gamma, \eta$ for the different test cases.

\subsection{Testing the one-species limit}

We compared the evolution of the electron density computed by the twospecies Vlasov-Poisson code with that obtained by a one-species simulation. The latter was performed by a similar kinetic code; the parameter $\alpha$ in (55) was taken as 1/1000 (corresponding to the two-species case 1) or 1/90000 (as in case 2).

The results are plotted on Figure 1. The divergence is hardly less rapid when the ions are heavier (case 2) than with the lighter ones (case 1); its characteristic time is of order $\bar{t}=2.38 \mathrm{~ns}$. The reason for this is probably the very strong electron Landau damping (a detailed numerical study based on spectral methods can be found in [27]) that prevails at this high temperature $(100 \mathrm{eV})$. In the one-species case, the electron density fluctuations are rapidly damped to their equilibrium values. On the other hand, in the two-species framework, the electrons are relaxed towards a slowly moving ion density pattern. On a larger time scale, the electrons appear to follow the motion of the ions: this is the quasi-neutral regime.
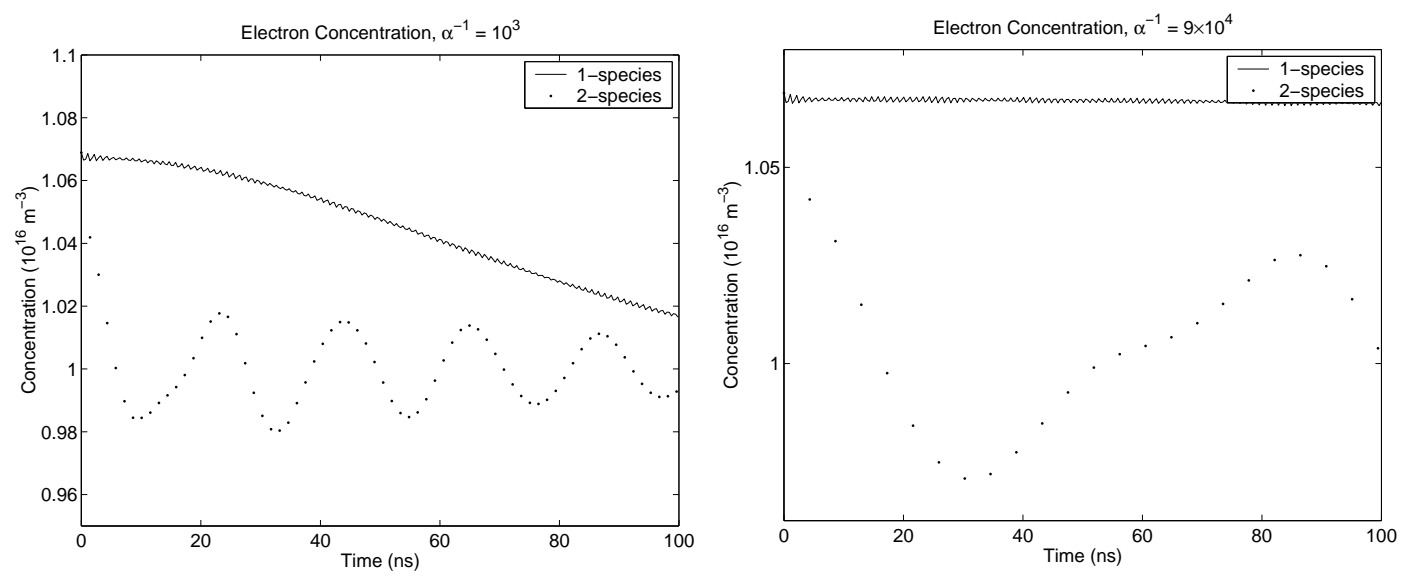

Fig. 1. Comparison of electron densities computed by one-species and two-species simulations. Left: case $1(\alpha=1 / 1000)$; right: case $2(\alpha=1 / 90000)$. Time scale: $\bar{t}=2.38 \mathrm{~ns}$. 


\subsection{Testing the quasi-neutrality}

So we were led to investigate the validity of the quasi-neutrality condition (42) and (43) for both species. As the code sets the additive constant of the potential by taking $\phi=0$ at the left extremity of the simulation box, Equation (43) is equivalent to:

$$
n_{i}(t, x)=n_{i}(t, 0) \exp \left[-\frac{\mu_{i} \phi(t, x)}{\theta_{i}(t, x)}\right] .
$$

To test (56), we made a semi-logarithmic plot of $\phi / T_{i}$ against the relative concentration $n_{i} / n_{i}(\cdot, 0)$. (We refer to this as the Boltzmann test.) The data were collected at seven selected points, equally spaced in the mesh.

The direct comparison of $n_{1}$ and $n_{2}$ gave good results in all cases (see Figures 2 and 3). The results of the Boltzmann test were more ambiguous. In the cases 1 and 2 (with the larger value of $\eta$ ) the electron data were fairly well aligned. For the ions, there is also some grouping, but it cannot correspond to (56), since this equation predicts a negative slope. However, as can be seen from Figure 3, the Boltzmann test failed completely in the cases 6 and 7, which intuitively correspond to a better quasi-neutrality. Nevertheless, as expected, the direct comparison appears much better than in the cases 1 and 2 .

\subsection{Testing the cold ion limit}

As explained in Section 5.1, we had to use a slightly different numerical method for the cold ion case. We implemented a two-species Euler-Poisson code with a non-characteristic WENO scheme; both species were represented by a twomoment system, i.e. treated as isothermal with a possibly vanishing temperature $T_{i}$.

The first task was to check that the non-characteristic method was not too inaccurate for allowing meaningful comparisons. To do so, we simulated a system made of isothermal electrons at $T_{1}=100 \mathrm{eV}$ and isothermal ions at $T_{2}=1 \mathrm{eV}$. This is clearly unphysical, but allows easy comparisons between the characteristic and the non-characteristic method. The results were almost undistinguishable on several periods; in other words, the non-characteristic method, though less good theoretically, is excellent for smooth solutions.

Thus we could reliably proceed to the numerical study of the cold ion limit. We simulated a system of isothermal electrons at $T_{1}=100 \mathrm{eV}$ and cold ions 

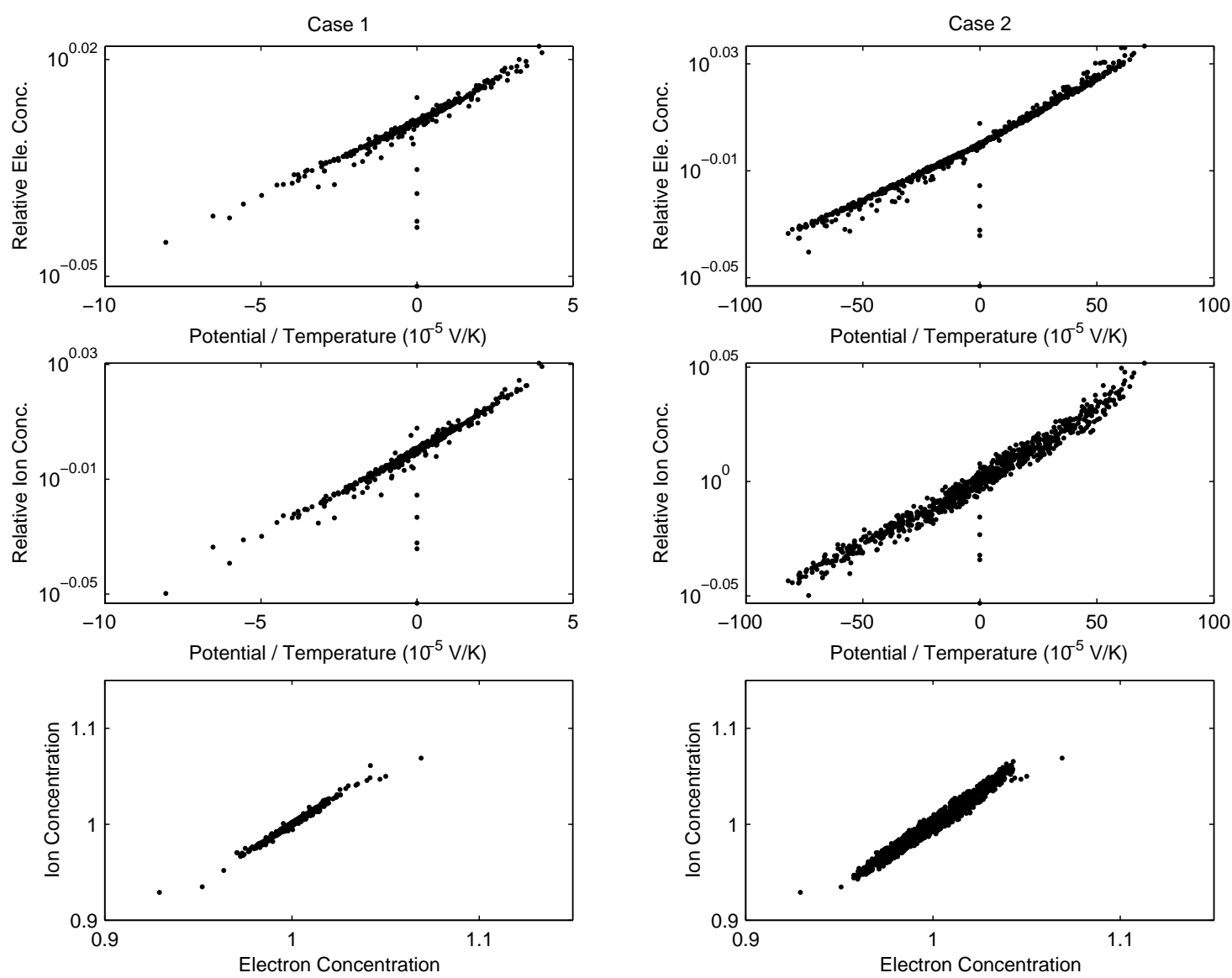

Fig. 2. The three quasi-neutrality tests, cases 1 and 2. Above: Boltzmann test for electrons. Middle: Boltzmann test for ions. Below: direct comparison of concentrations.

at $T_{2}=0 \mathrm{eV}$ by the non-characteristic code. The parameter $\gamma$ was taken as $1 / 100$ or $1 / 900$, which allowed direct comparisons with the cases 1 to 4 of the characteristic WENO simulations. Figure 4 shows the influence of the ion temperature, hence of the parameter $\beta$. The cold ion limit is defined by $\alpha \rightarrow 0$; when $\gamma$ is fixed, this is equivalent to $\beta \rightarrow 0$. We remark that adiabatic simulations at $\beta=1 / 100$ stand very close to cold ion simulations on at least two periods. The cold ion limit thus appears as a valid approximation when $\beta \ll 1$.

\subsection{Testing the thermodynamic hypotheses}

In order to check the thermodynamic hypotheses that founded the fluid derivation, viz. that the ions were adiabatic and the electrons were isothermal, we calculated the fluid quantities by a kinetic simulation. Then we performed three types of tests to assess these hypotheses. 

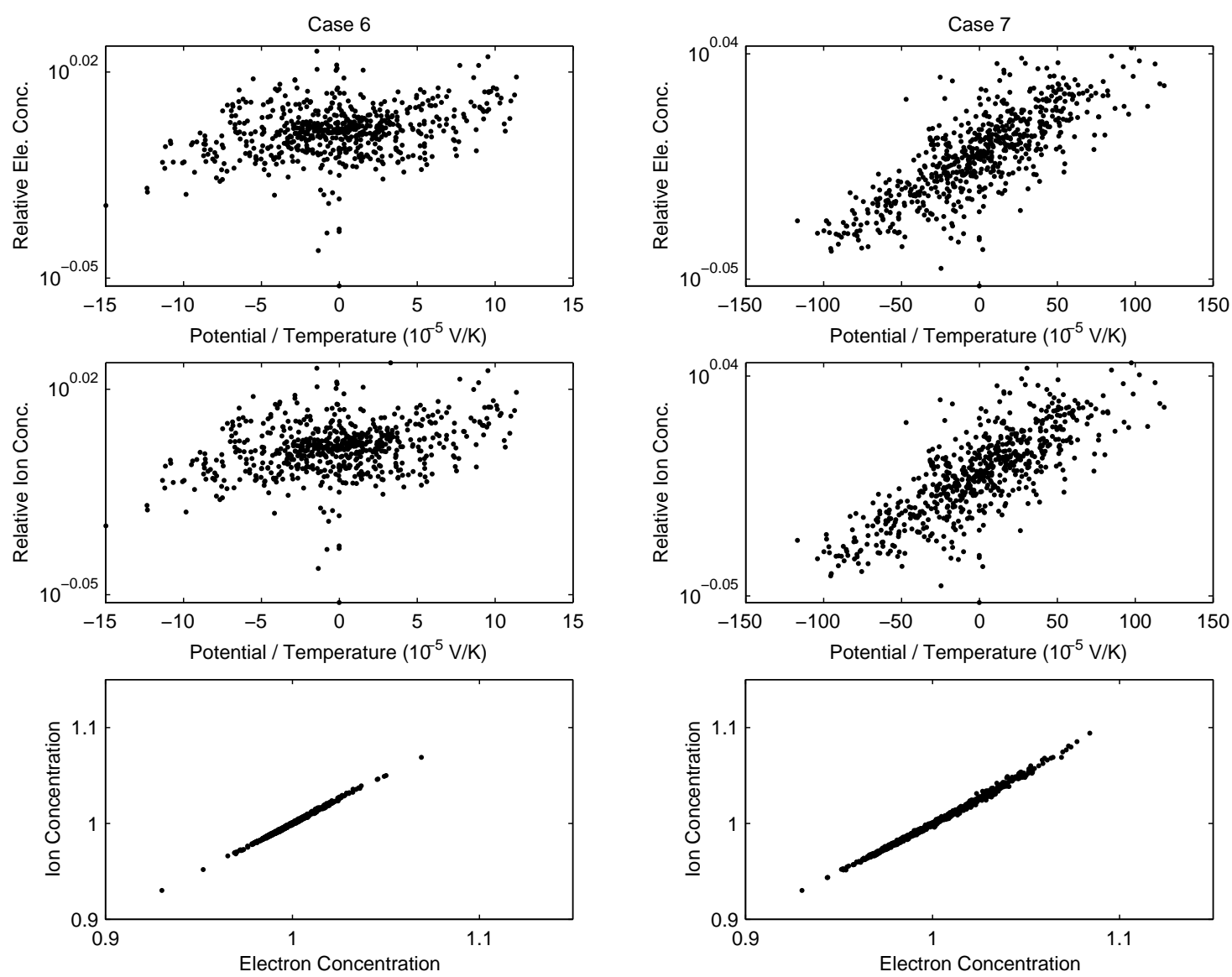

Fig. 3. The three quasi-neutrality tests, cases 6 and 7. Above: Boltzmann test for electrons. Middle: Boltzmann test for ions. Below: direct comparison of concentrations.

First, we recorded the time evolution of the temperatures of both species, at our seven points. Figure 5 shows this evolution at one of these points. We see that, while the amplitude of the relative variation of $T_{2}$ is of order $\varepsilon=0.05$, the amplitude of the electron temperature is much smaller. Thus, the electrons can be reasonably considered as isothermal for any $\gamma \leq 1 / 100$.

Then, we compared the divergence of the heat flux (i.e. $\partial_{x} K_{i}$ ), with the divergence of the energy-pressure flux (i.e. $\partial_{x}\left\{\left(w_{i}+p_{i}\right) u_{i}\right\}$, for $i=1$ and 2 . If the former is negligible when compared to the latter, the species under consideration can be seen as adiabatic.

Figure 6 shows that this is never the case for the electrons. As for the ions, it seems that they can be reasonably considered as adiabatic for $\beta=1 / 100$, but not for $\beta=1 / 10$ : in this case, the two flux divergences are of the same order of magnitude.

The last test was to plot a density-pressure diagram. In logarithmic scale, 

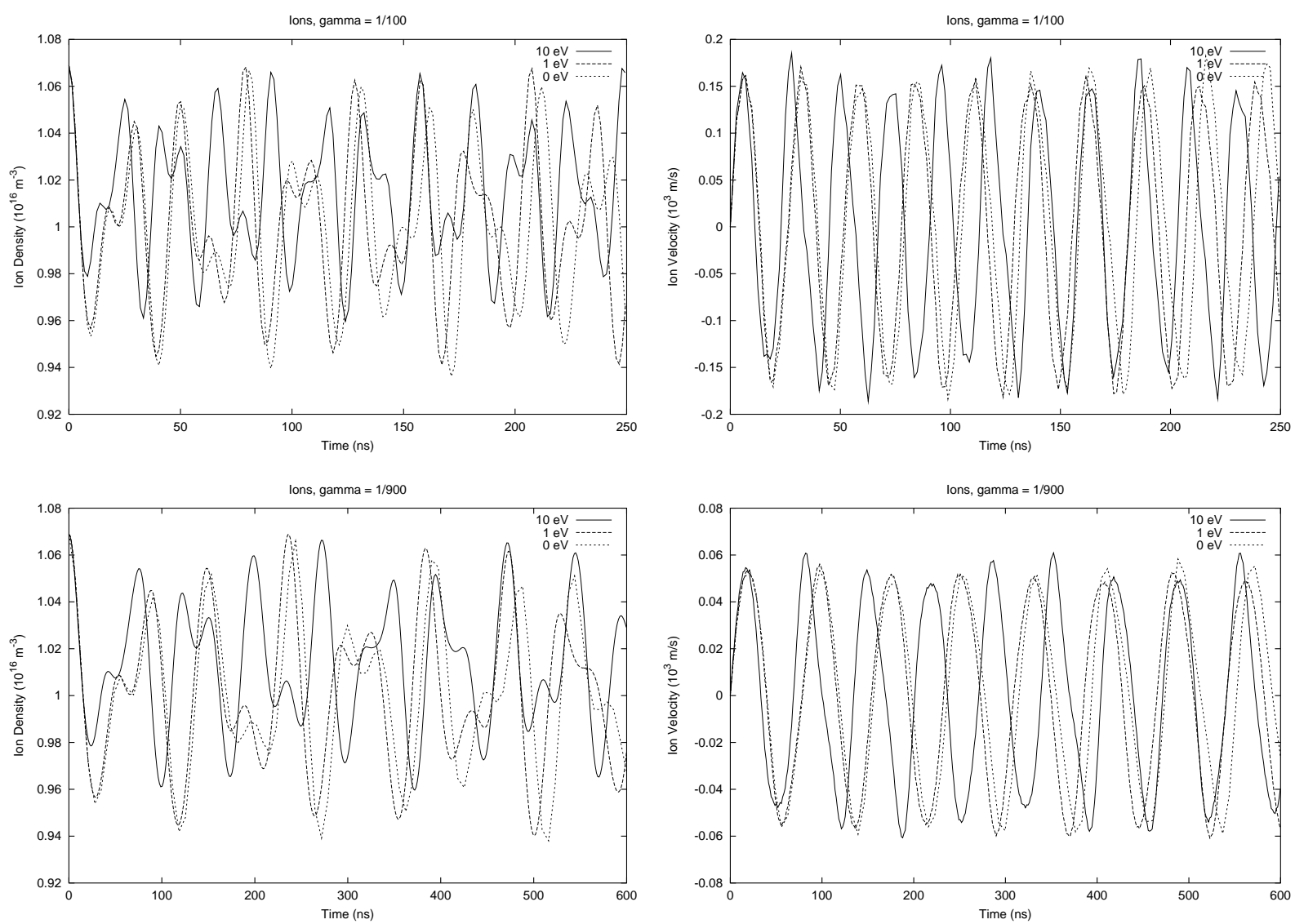

Fig. 4. Comparison of finite and zero temperature simulation for ion density (left column) and ion velocity (right column). The curves are labelled by the ion temperature. Time scale: $\bar{t}=2.38 \mathrm{~ns}$.

the pressure as a function of density should appear as a straight line for an isothermal species, and as a set of parallel straight lines for an adiabatic one. (Remember that the constant $A$ in the pressure law (20) depends on the trajectory.) Using the values recorded at all our seven points, we got the results shown on Figure 7.

These confirm the two previous tests. The electron density and pressure are grouped along a straight line in all test cases. As for the ions, the parallel structure is rather apparent in the case $\beta=1 / 100$, but much less for $\beta=1 / 10$.

The three previous tests have also been performed for the cases 5 to 8 . All the results were qualitatively similar. 

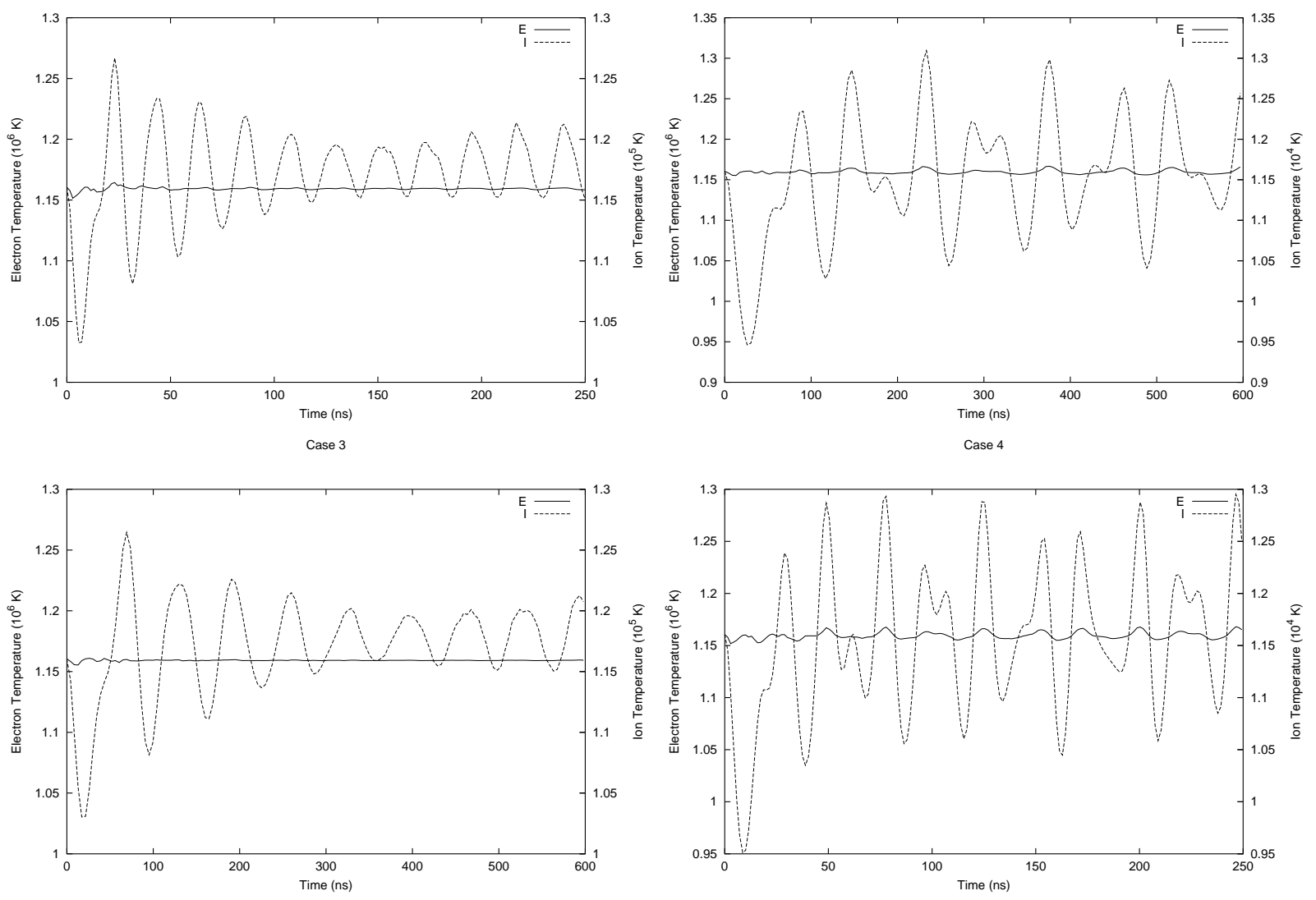

Fig. 5. Evolution of temperature with time for electrons (E, solid line) and ions (I, dashed line). Time scale: $\bar{t}=2.38 \mathrm{~ns}$.

\subsection{Testing the fluid approximation}

Then, we compared the results of fluid and kinetic simulations. To do so, we plotted the density and fluid velocity at one point. For the ions, fluid and kinetic simulations are qualitatively similar. The frequences are of the same order (as can be checked by a Fourier analysis) and the two curves coincide more or less during the first period. The quality of the fluid approximation seems much better for $\beta=1 / 100$, and the fluid simulations remain remarkably precise for several periods. Clearly, the main cause of the divergence of the curves is the Landau damping for both species, ignored by the fluid model. For the same reason, the divergence in the fluid-kinetic comparison for electrons is faster, so that the fluid approximation becomes bad in time scales shorter than the period of the wave. We have checked that this is not a numerical artifact, by a refinement study in all variables.

Another interesting test is to compare the distribution function (DF) with the local Maxwellian having the same first three moments (density, fluid velocity 
and temperature). So we define the deviation of the DF as:

$$
\delta f_{i}(t, x, v)=f_{i}(t, x, v)-n_{i}(t, x) \mathcal{M}_{\theta_{i}(t, x)}\left(v-u_{i}(t, x)\right)
$$

and we shall refer to $f_{i}$ itself as the brute DF. We plotted snapshots of $f_{2}$ and $\delta f_{2}$, for cases 1 and 2 on Figures 9-12. The same level set is always drawn in the same colour in all the snapshots within a given figure. Units are fairly arbitrary, but are the same for $f$ and $\delta f$ : so, one sees that the typical value of $\delta f_{2}$ is less than one tenth of $f_{2}$ : the departure from Maxwellian equilibrium, though small, is not insignificant. Rather than in its magnitude, the difference between the "good" case 2-where the fluid approximation appears reasonable - and the "bad" case 1 - where it is inacceptable - lies in the structure of $\delta f_{2}$.

One observes the well-known "filamentation" phenomenon. When small enriched or depleted regions appear, their contours are deformed by the Vlasov dynamic: the "far side" (high velocity in absolute value) moves faster than the "near side", closer to the middle of the phase-space diagram. This causes those regions to be stretched thinner and thinner; if the phenomenon intensively repeats itself, steep gradients of $f$ can appear in the $v$-direction; then more complex dynamical phenomena may rotate them to any direction. This occurs especially when particules are trapped and detrapped in the potential wells of the waves, resulting in a folding-stretching of the phase-space contours. One clearly observes that the filamentation is more important in case 1, and this purely kinetic phenomenon is probably the good criterion of the departure from fluid behaviour.

As for the electrons, their DF appears to be very close to a Maxwellian: $\left|\delta f_{1}\right| / f_{1} \leq 2 \%$. No clear structure appears in $\delta f_{1}$, which looks very noisy. This tends to confirm that the magnitude of the deviation from the Maxwellian is not the only measurement of the departure from fluid behaviour.

Once more, these comparisons and tests have also been carried out for the cases 5 to 8 , and the conclusions are similar.

\section{Summary and discussion}

In this paper, we performed an extensive numerical study of certain approximations which are of general use both in the theoretical and computational study of challenging problems of plasma physics, such as turbulence and anomalous transport in tokamak plasmas. Namely, we were interested mainly in the fluid and quasi-neutral approximations, and secondarily in one-species 
and zero-temperature limits. To assess the validity of these approximations as such, they were studied in the context of a simplified model which, however, contains all of them: the one-dimensional, two-species Vlasov-Poisson system.

It appears that the fluid approximation is adequate even in the absence of collisions (i.e. on a time scale quite smaller than the collision time), provided the characteristic speed of the phenomenon under consideration is different enough from the thermal velocities of both species. The degree of accuracy agrees with the "confidence rate" of Table 1, which suggests that the divergence between both models is mainly caused by Landau damping. This result is of great importance from a computational point of view, because it may justify the use of the much less costly fluid simulations in some cases. But there is also a physical implication: the magnitude of the deviation from Maxwellian equilibrium may not be the one and only criterion for judging the departure from fluid behaviour. More complex criteria, involving derivatives of the distribution function and thus measuring the filamentation phenomenon, should probably be used.

To find the good quasi-neutrality equation has always been the hardship of modellers; and the derivation of an asymptotic quasi-neutral dynamic seems out of reach under most circumstances. Surprisingly, the "brute" equation (42) appears much more exact when one tends to neutrality, than the seemingly more sensible condition (43). Indeed, Eq. (43) breaks down for the ions, as expected; but also for the electrons when the parameter $\eta$, which measures the degree of neutrality, goes to 0. This seems rather annoying since Eq. (43) is very widely used. So far, we have no satisfactory explanation for this finding, which has still to be confirmed by other simulations. Assuming that (43) holds for the electrons only is consistent with the basic physical features of our model - at least under some extra assumptions that are satisfied in all our test cases. A numerical artifact is unlikely. The small $\eta$ simulations have excellent qualitative properties and behave as expected in all the other tests. Moreover, the overall efficiency of the code does not depend on $\eta$. We are in front of a really challenging modelling problem: it seems that one has to use two different, and incompatible, quasi-neutrality equations according to the degree of neutrality expected.

The cold ion approximation - which is really a particular case of fluid approximation - appears fairly accurate when the thermal velocities of both species are several orders of magnitude apart. But, from a computational point of view, the lack of hyperbolicity of this model precludes the use of the most accurate hyperbolic solvers. This could be a drawback if highly irregular (e.g. turbulent) behaviours were expected.

Finally, the one-species model seems highly inaccurate except on the shortest 
time scales, for instance that of the electron plasma oscillations.

The assumptions lying beneath these approximations are of thermodynamic nature. Thus, their validity should not be affected when carrying over to complex, three-dimensional geometries. Similarly, the results of this study could be used even if new physical phenomena (turbulence, magnetic field) are added to the model, unless the extra physics demonstrably interferes with those assumptions.

We also hope that these simulations have demonstrated the potentialities of WENO schemes, which had been already used both for ordinary gas dynamics [26] and for some kinetic problems, such as those arising in semiconductor physics $[24,25]$. Plasma physics, both in the kinetic and fluid approaches, is a natural field of application for these powerful, accurate and flexible methods.

\section{Acknowledgements}

Part of this work was done when S.L. was visiting J.A.C. at the University of Granada. S.L. is indebted to the European Research and Training Network "Hyperbolic and Kinetic Equations" (HYKE) for its support during this visit, and to the University of Granada for time allocation on the Silicon Graphics Origin 3400 supercomputer. J.A.C. acknowledges the support from the Spanish DGI-MCYT/FEDER project BFM2002-01710.

\section{References}

[1] W. Dorland, G.W. Hammett, Gyrofluid turbulence models with kinetic effects, Phys. Fluids B, 5, 812-835 (1993).

[2] X. Garbet R.E. Waltz, Action at distance and Bohm scaling of turbulence in tokamaks, Phys. Plasmas, 3, 1898-1907 (1996).

[3] G. Manfredi, M. Ottaviani, Gyro-Bohm Scaling of Ion Thermal Transport from Global Numerical Simulations of Ion-Temperature-Gradient-Driven Turbulence, Phys. Rev. Lett., 79, 4190-4193 (1997).

[4] S.E. Parker, W.W. Lee, R.A. Santoro, Gyrokinetic simulation of ion temperature gradient driven turbulence in 3D toroidal geometry, Phys. Rev. Lett., 71, 2042-2045 (1993).

[5] R.D. Sydora, V.K. Decyk, J.M. Dawson, Fluctuation-induced heat transport results from a large global 3D toroidal particle simulation mode, Plasma Phys. Control. Fusion, 38, A281-A294 (1996). 
[6] G. Depret, X. Garbet, P. Bertrand, A. Ghizzo, Trapped ion driven turbulence in tokamak plasmas, Plasma Physics and Controlled Fusion, 42, 949-971 (2000).

[7] M. Brunetti, V. Grandgirard, S. Allfrey, A. Bottino, P. Bertrand, Comparisons between Semi-Lagrangian Drift-Kinetic Code and PIC Code Simulation for ITG Studies, 29 EPS conference, Montreux, 17-21 June 2002.

[8] N. Mattor, Can Landau-fluid models describe nonlinear Landau damping? Phys. Fluids B, 4, 3952-3961 (1992).

[9] P. Bertrand, M.R. Feix, Nonlinear electrons plasmas oscillations: the "water bag model", Phys. Lett. A, 28, 68-69 (1968).

[10] M. Gros, P. Bertrand, M.R. Feix, Connection between hydrodynamic, water bag and Vlasov models, Plasma Physics, 20 1075-1080 (1978).

[11] C. Cercignani, R. Illner, M. Pulvirenti, The mathematical theory of dilute gases, Applied Mathematical Sciences 106, Springer, New York, 1994.

[12] C.W. Shu, Essentially non-oscillatory and weighted essentially nonoscillatory schemes for hyperbolic conservation laws, in Advanced Numerical Approximation of Nonlinear Hyperbolic Equations, B. Cockburn, C. Johnson, C.W. Shu and E. Tadmor (Editor: A. Quarteroni), Lecture Notes in Mathematics, volume 1697, Springer, 1998, pp. 325-432.

[13] S. Cordier, Global solutions to the isothermal Euler-Poisson plasma model, Appl. Math. Lett. 8 19-24 (1995).

[14] S. Cordier, P. Degond, P.A. Markowich, C. Schmeiser, Travelling wave analysis and jump relations for Euler-Poisson model in the quasineutral limit, Asymptotic Anal. 11 209-240 (1995).

[15] S. Cordier, P. Degond, P.A. Markowich, C. Schmeiser, Travelling wave analysis of an isothermal Euler-Poisson model, Ann. Fac. Sci. Toulouse Math. 5 599-643 (1996).

[16] S. Cordier, E. Grenier, Quasineutral limit of an Euler-Poisson system arising from plasma physics, Comm. P.D.E. 25 1099-1113 (2000).

[17] R.J. Goldston, P.H. Rutherford, Introduction to plasma physics, Institute of Physics Publishing, Bristol, 1995.

[18] Y. Brenier, Convergence of the Vlasov-Poisson system to the incompressible Euler equations, Comm. P. D. E. 25 737-754 (2000).

[19] L.D. Landau, On the vibrations of the electronic plasma, J. Phys. X 25-34 (1946).

[20] F. Filbet, E. Sonnendrücker, Comparison of Eulerian Vlasov Solvers, Comp. Phys. Comm. 150 247-266 (2003).

[21] S. Gottlieb, C.W. Shu, Total variation diminishing Runge-Kutta schemes, Math. Comp. 67 73-85 (1998). 
[22] G. Jiang, C.W. Shu, Efficient implementation of weighted ENO schemes, J. Comput. Phys. 126 202-228 (1996).

[23] C.W. Shu, S. Osher, Efficient implementation of essentially non-oscillatory shock capturing schemes, J. Comput. Phys. 77 439-471 (1988).

[24] C. Cercignani, I.M. Gamba, J.W. Jerome, C.W. Shu, Device benchmark comparisons via kinetic, hydrodynamic, and high-field models, Comput. Meth. Appl. Mech. Engin. 181 381-392 (2000).

[25] J.A. Carrillo, I. Gamba, C.W. Shu, Computational macroscopic approximations to the 1-D relaxation-time kinetic system for semiconductors, Physica D $\mathbf{1 4 6}$ 289-306 (2000).

[26] C.W. Shu, T.A. Zang, G. Erlebacher, D. Withaker, S. Osher, High-order ENO schemes applied to two- and three-dimensional flows, Appl. Numer. Math. 9 45-71 (1992).

[27] T. Zhou, Y. Guo, C.W. Shu, Numerical study on Landau damping, Physica D 157 322-333 (2001). 

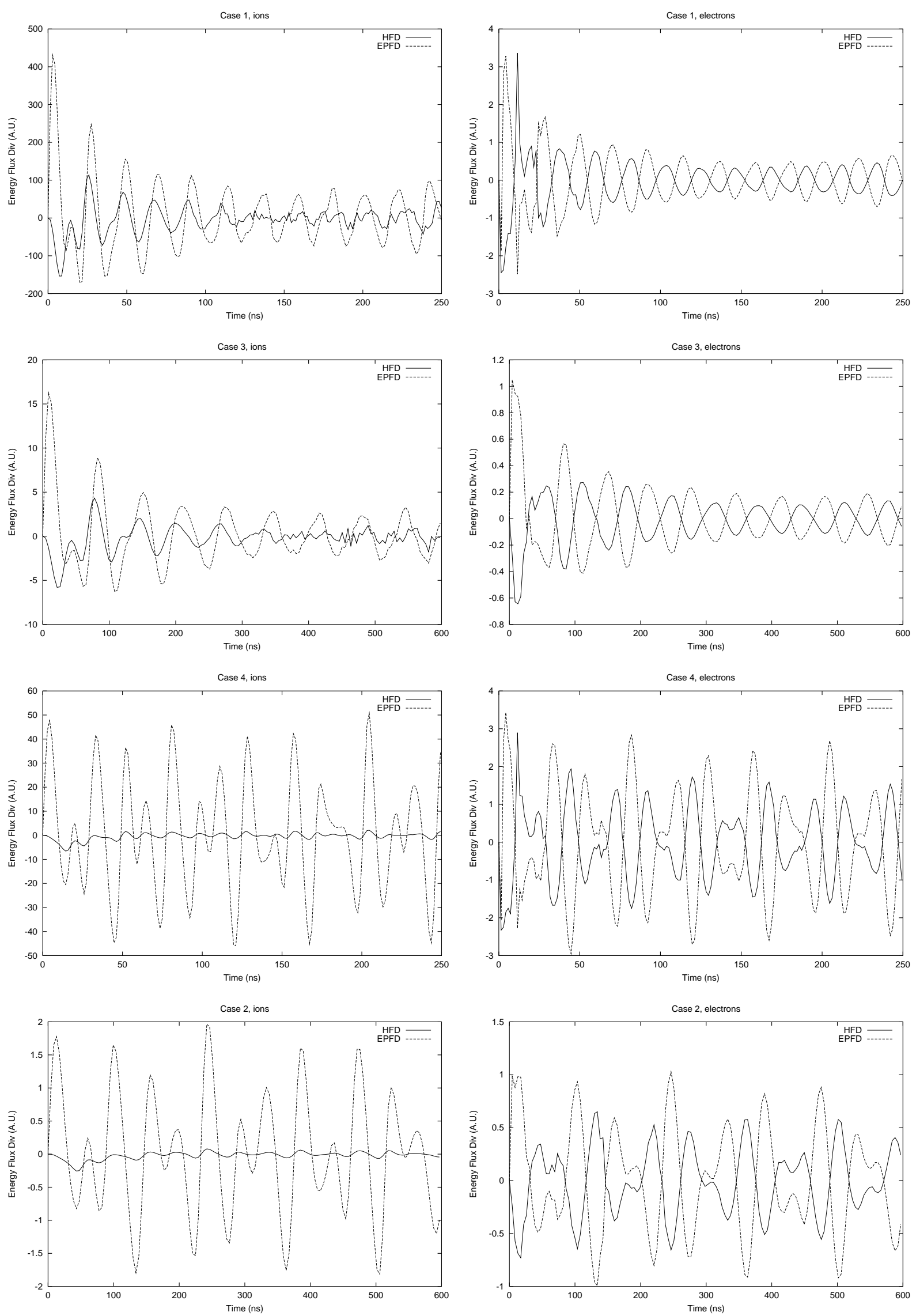

Fig. 6. Comparison of heat flux divergence (HFD) and energy-pressure flux divergence (EPFD) for the ions (left column) and the electrons (right column). 

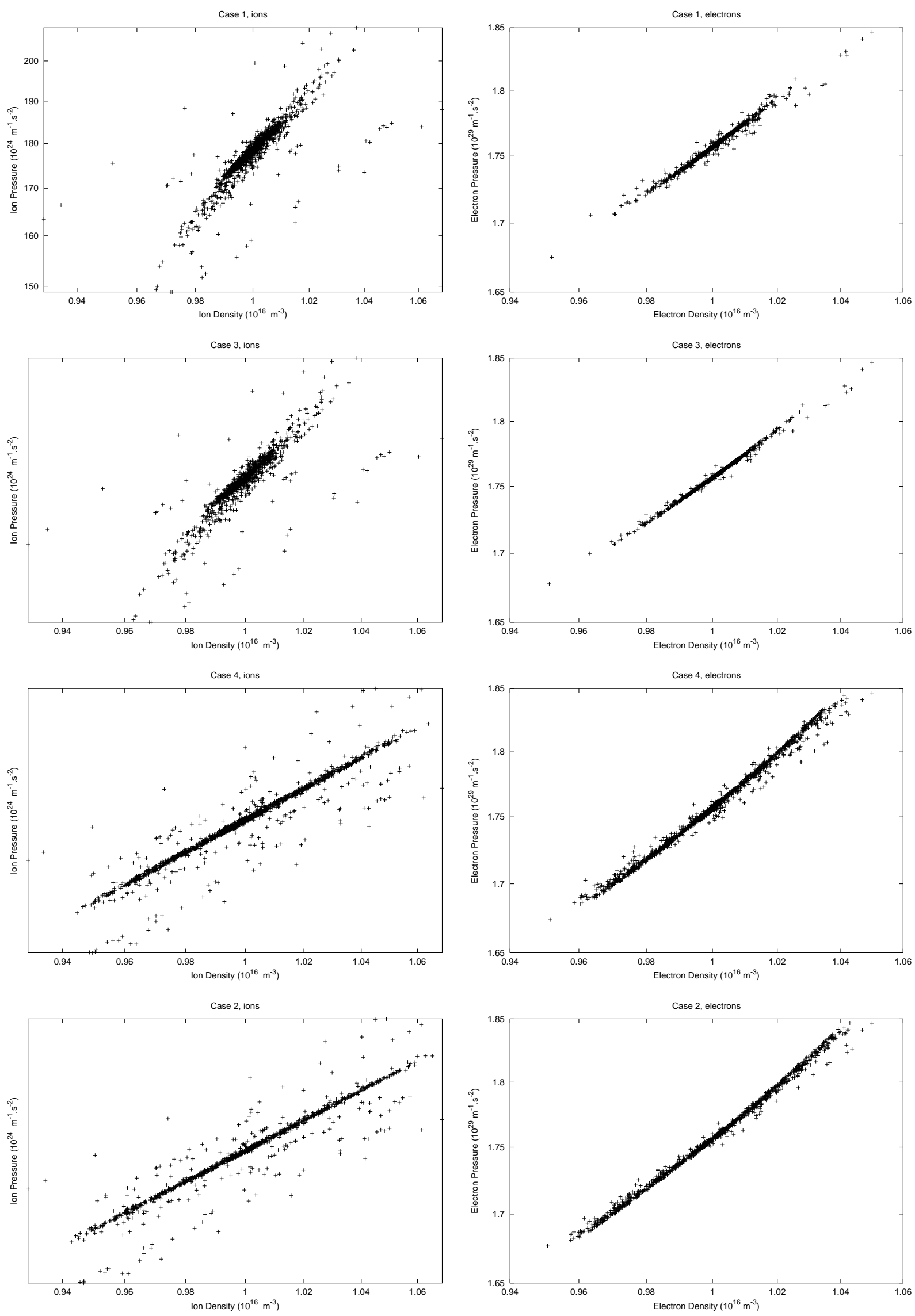

Fig. 7. Density-pressure diagram for the ions (left column) and the electrons (right column). 

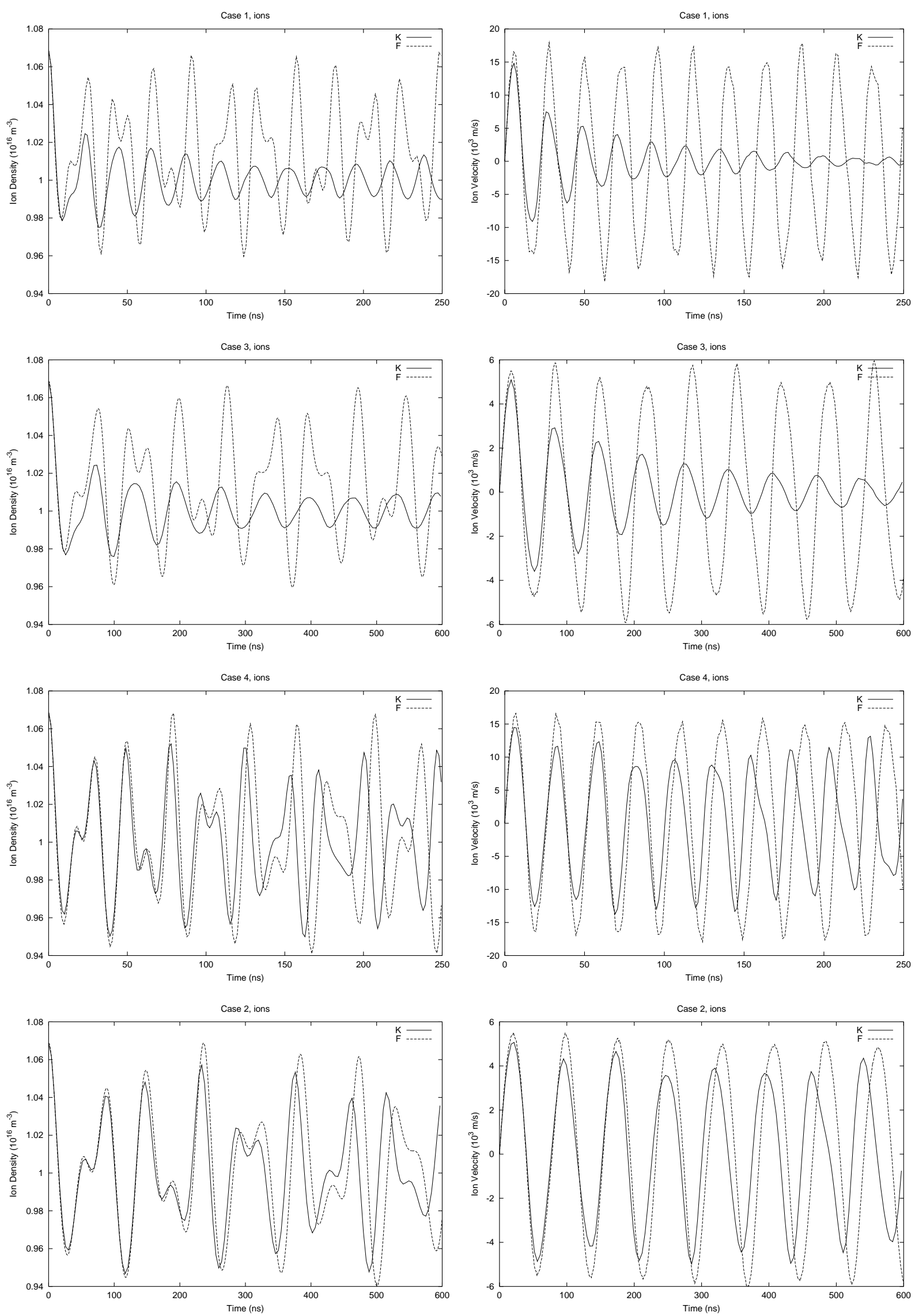

Fig. 8. Comparison of kinetic (K, solid line) and fluid (F, dashed line) simulations for ion density (left column) and ion velocity (right column). 

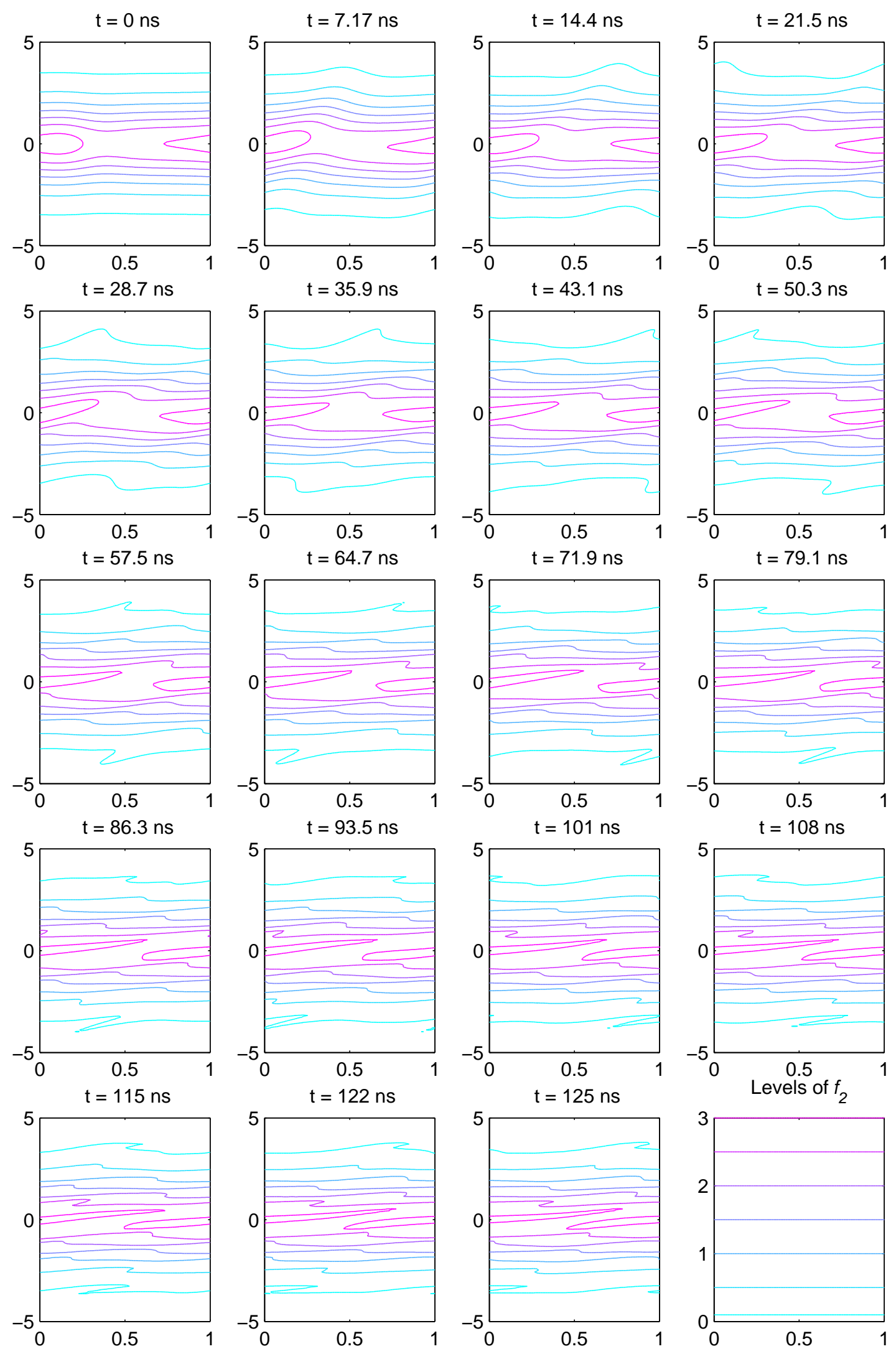

Fig. 9. Snapshots of the brute ion DF, case 1. 

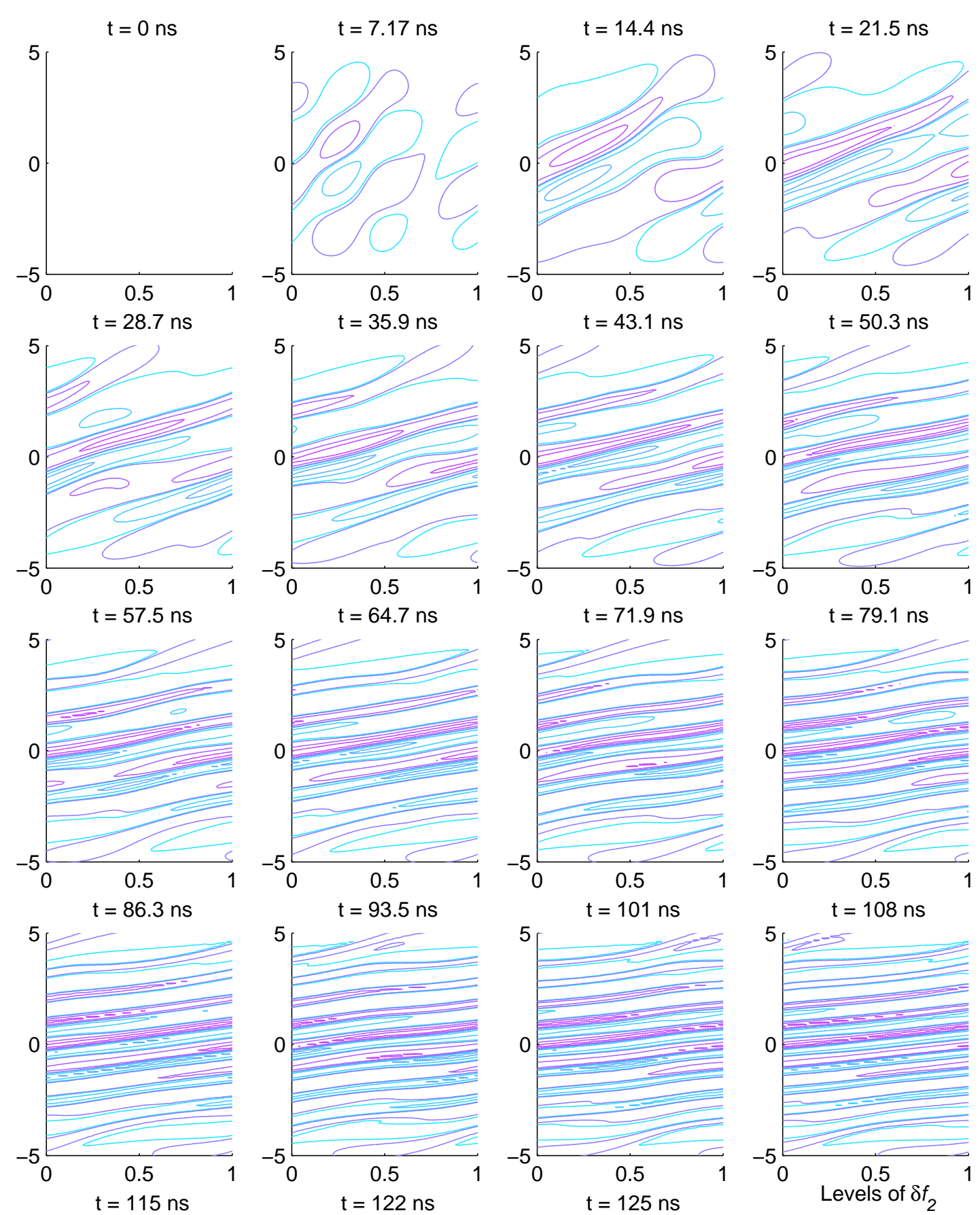

$\mathrm{t}=108 \mathrm{~ns}$
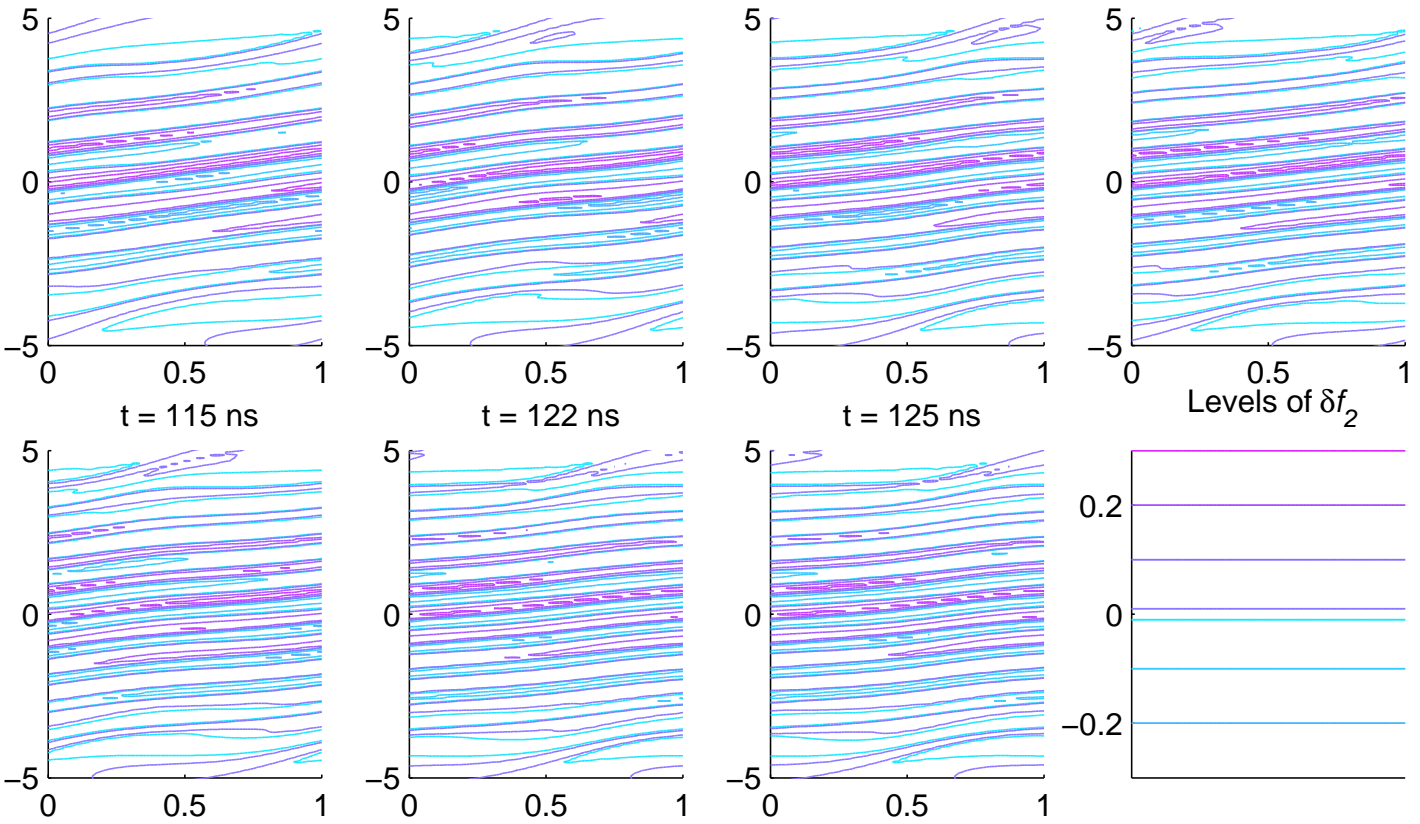

Fig. 10. Snapshots of the deviation of the ion DF, case 1. 

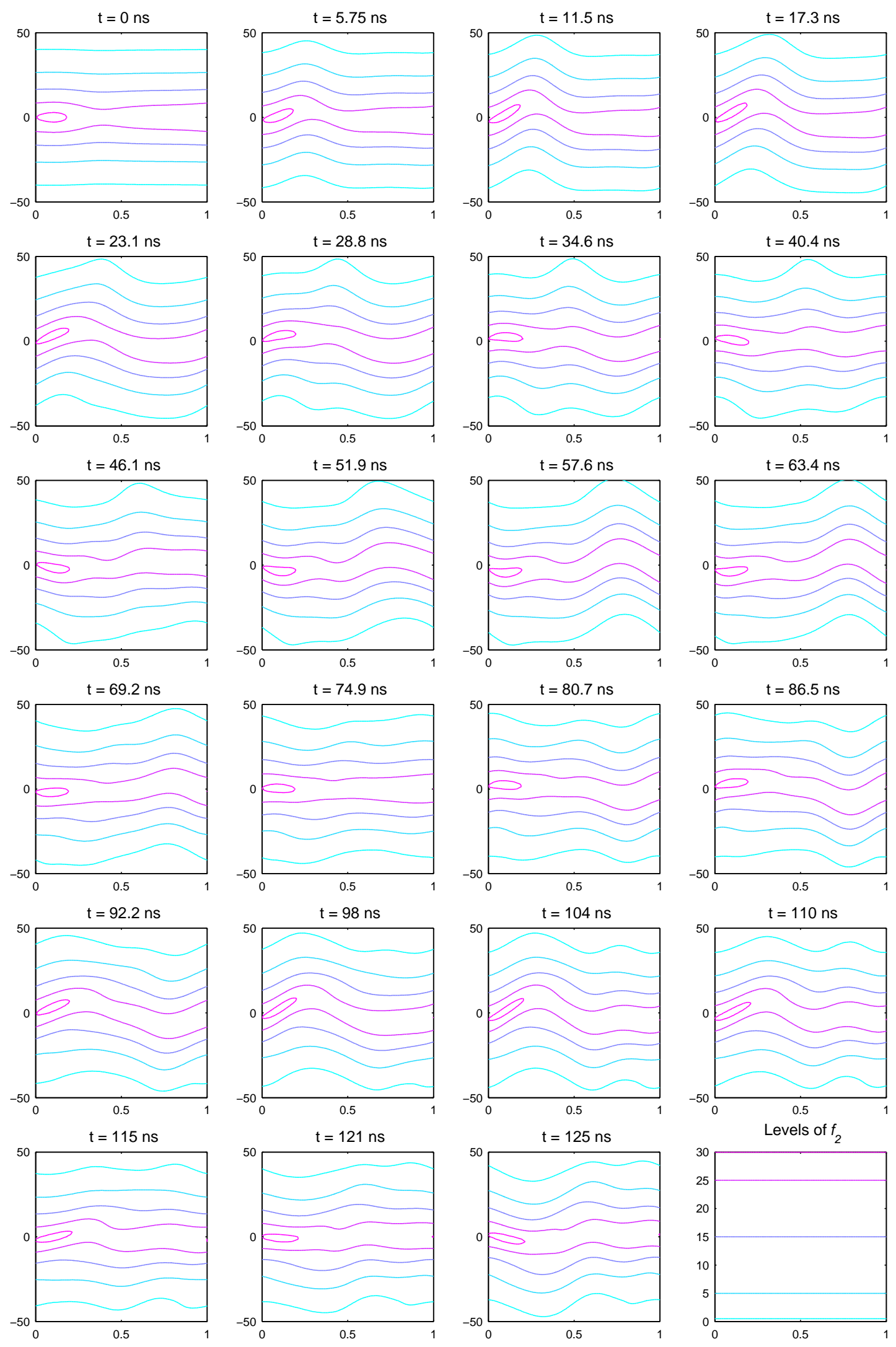

Fig. 11. Snapshots of the brute ion DF, case 2 . 

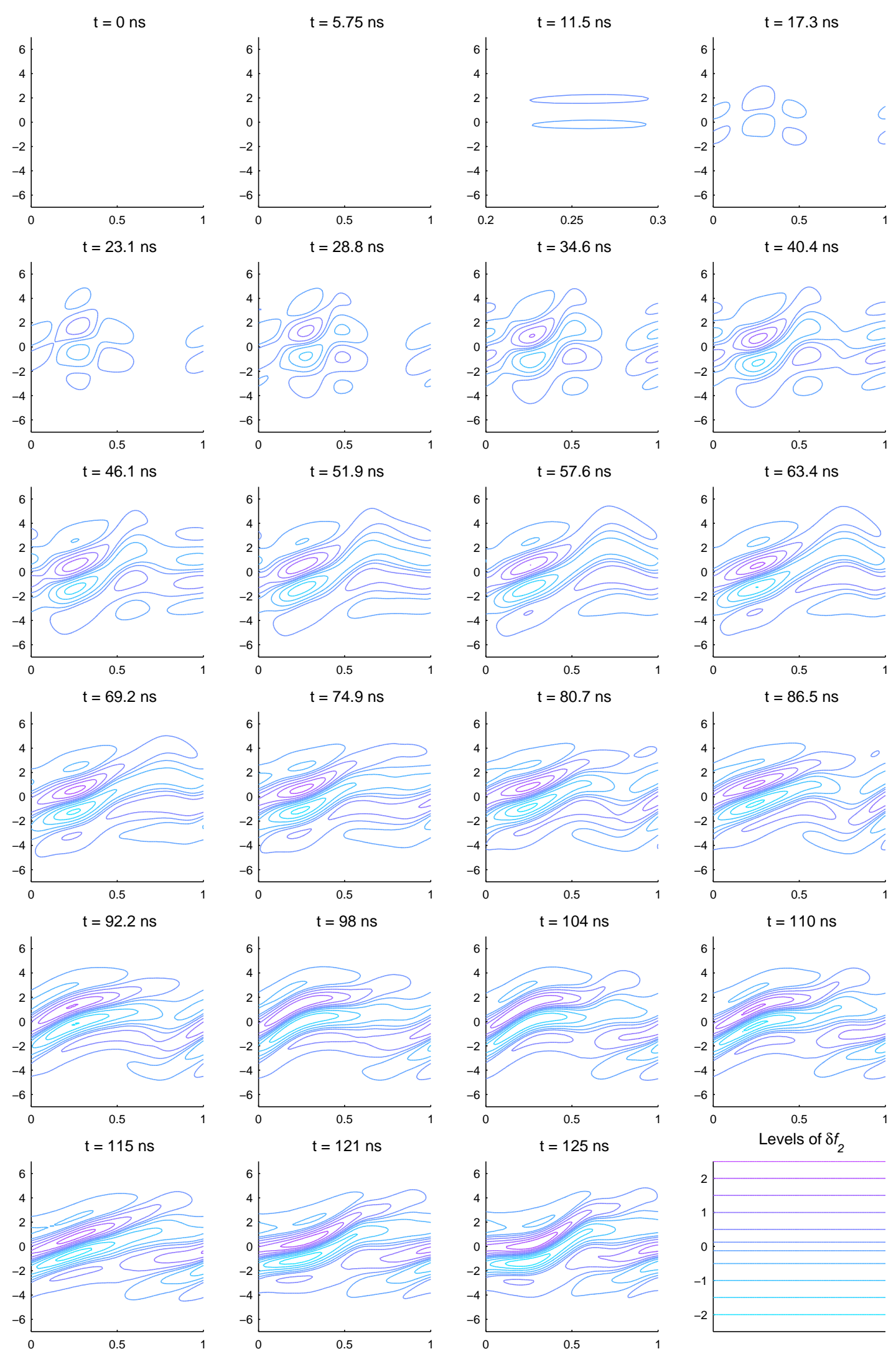

Fig. 12. Snapshots of the deviation of the ion DF, case 2 . 


\section{A The Boltzmannian electron regime}

We consider a linear potential regime which is a little more general than in Section 3.3, namely:

- $\phi \ll \theta_{1}$ and $\theta_{1} \simeq 1$ (in rescaled units);

- Eq. (43) is satisfied for the electrons; with the previous assumption this gives $n_{1}(x)=(1+\phi(x)) n_{1}(0)$;

- $n_{2}(x)$ is a given function, endowed with three derivatives in $x$ that are integrable on $(0,1)$.

The last assumption is essentially technical. It holds e.g. in the kinetic framework, if we suppose that $f_{2}(0, x, v)$ is thrice differentiable. This property will be conserved with time and carry over to $n_{2}$. The first condition is satisfied in all our test cases, see Figures 2, 3 and 5.

Assuming $Z=1$, or redefining $n_{2}$ as $Z$ times the actual ion density, we find that the potential is solution to

$$
-\eta^{2} \phi^{\prime \prime}(x)+k^{2} \phi(x)=n_{2}(x)-k^{2}
$$

where, this time, $k^{2}=n_{1}(0)$. (Here, too, we forget the $t$-dependence, and we denote by ' the $x$-derivative.) Using the "variation of constants" formula and a bit of trigonometry, we find that the solution to (A.1) can be written:

$$
\begin{aligned}
\phi(x)= & \frac{1}{k \eta}\left[\frac{\sinh \lambda(1-x)}{\sinh \lambda} \int_{0}^{x} \sinh \lambda y\left(n_{2}(y)-k^{2}\right) \mathrm{d} y\right. \\
& \left.+\frac{\sinh \lambda x}{\sinh \lambda} \int_{x}^{1} \sinh \lambda(1-y)\left(n_{2}(y)-k^{2}\right) \mathrm{d} y\right]
\end{aligned}
$$

where $\lambda=k / \eta$. The periodicity condition is given by $\phi^{\prime}(0)=\phi^{\prime}(1)$; after some calculations, we obtain that this is equivalent to:

$$
\int_{0}^{1} w_{\lambda}(y)\left(n_{2}(y)-k^{2}\right) \mathrm{d} y=0, \quad \text { where: } \quad w_{\lambda}(y)=\frac{\cosh \left\{\lambda\left(y-\frac{1}{2}\right)\right\}}{\cosh (\lambda / 2)}
$$

It is a lengthy, but interesting exercise, to check that the global neutrality condition is also given by (A.3). 
Hence, for a given value of $\eta$, Eq. (A.1) admits a physically relevant solution iff $\lambda$ is solution to:

$$
F(\lambda) \stackrel{\text { def }}{=} \int_{0}^{1} w_{\lambda}(y) n_{2}(y) \mathrm{d} y=(\lambda \eta)^{2} \int_{0}^{1} w_{\lambda}(y) \mathrm{d} y=2 \eta^{2} \lambda \tanh \frac{\lambda}{2} .
$$

The function $F(\lambda)$ enjoys the following properties:

- As the functions $n_{2}$ and $w_{\lambda}$ are positive, $F(\lambda) \geq 0$.

- $w_{0}(x)=1$, hence $F(0)=\int_{0}^{1} n_{2}(x) \mathrm{d} x=1$, whatever the form of $n_{2}$.

- For a given $x \in(0,1), w_{\lambda}(x)$ is strictly decreasing with $\lambda$ and tends to zero as $\lambda \rightarrow \infty$; hence, $F(\lambda)$ is a strictly decreasing function which goes to zero, by Lebesgue's theorem.

- Using an integration by parts and a similar argument as above:

$$
F(\lambda) \sim 2 n_{2}(0) / \lambda \quad \text { when } \lambda \rightarrow \infty
$$

Now, we rewrite (A.4) as:

$$
G(\lambda) \stackrel{\text { def }}{=} \frac{F(\lambda)}{2 \lambda \tanh (\lambda / 2)}=\eta^{2}
$$

From the above properties of $F$, it follows that $G(\lambda)$ is a strictly decreasing function, which tends to infinity as $\lambda \rightarrow 0$ and to 0 as $\lambda \rightarrow \infty$. Hence, (A.6) admits a unique solution $\lambda(\eta)$ for any value of $\eta>0$ : our model is consistent. Clearly, $\lambda(\eta)$ is a decreasing function of $\eta$, and $\lambda(\eta) \rightarrow \infty$ when $\eta \rightarrow 0$. If $\eta$ is small enough, $\lambda(\eta)$ is large enough and we can replace $F(\lambda)$ and $\tanh (\lambda / 2)$ by their equivalent in (A.6):

$$
\frac{2 n_{2}(0) / \lambda}{2 \lambda} \sim \eta^{2}, \quad \text { i.e.: } \lambda(\eta) \sim \eta^{-1} \sqrt{n_{2}(0)}, \text { and: } k(\eta)=\eta \lambda(\eta) \sim \sqrt{n_{2}(0)} .
$$

We are now able to derive the asymptotic behaviour of $\phi$ and $n_{1}$ when $\eta \rightarrow 0$. Performing three successive integrations by parts in (A.2), we obtain

$$
\begin{aligned}
k \eta \phi(x)= & \frac{1}{\lambda}\left[n_{2}(x)-k^{2}-\frac{\sinh \lambda(1-x)}{\sinh \lambda}\left(n_{2}(0)-k^{2}\right)-\frac{\sinh \lambda x}{\sinh \lambda}\left(n_{2}(1)-k^{2}\right)\right] \\
& +\frac{1}{\lambda^{3}}\left[n_{2}^{\prime \prime}(x)-\frac{\sinh \lambda(1-x)}{\sinh \lambda} n_{2}^{\prime \prime}(0)-\frac{\sinh \lambda x}{\sinh \lambda} n_{2}^{\prime \prime}(1)\right] \\
& +\frac{1}{\lambda^{3}}\left[-\frac{\sinh \lambda(1-x)}{\sinh \lambda} \int_{0}^{x} \cosh \lambda y n_{2}^{\prime \prime \prime}(y) \mathrm{d} y\right.
\end{aligned}
$$




$$
\left.+\frac{\sinh \lambda x}{\sinh \lambda} \int_{x}^{1} \cosh \lambda(1-y) n_{2}^{\prime \prime \prime}(y) \mathrm{d} y\right] .
$$

Let us call $R(x)$ the last bracket. We bound it as:

$$
\begin{aligned}
|R(x)| \leq & \frac{\sinh \lambda(1-x)}{\sinh \lambda} \cosh \lambda x \int_{0}^{x}\left|n_{2}^{\prime \prime \prime}(y)\right| \mathrm{d} y \\
& +\frac{\sinh \lambda x}{\sinh \lambda} \cosh \lambda(1-x) \int_{x}^{1}\left|n_{2}^{\prime \prime \prime}(y)\right| \mathrm{d} y \\
= & \frac{\sinh \{\lambda(1-x)+\lambda x\}}{\sinh \lambda} \int_{0}^{1}\left|n_{2}^{\prime \prime \prime}(y)\right| \mathrm{d} y=O(1) .
\end{aligned}
$$

On the other hand, the terms $\sinh \{\lambda(1-x)\} / \sinh \lambda$ and $\sinh \{\lambda x\} / \sinh \lambda$ are exponentially decreasing when $\lambda \rightarrow \infty$ and $0<x<1$. Finally, we have:

$$
k^{2} \phi(x)=n_{2}(x)-k^{2}+O\left(\eta^{2}\right) \quad \text { and } \quad n_{1}(x)=k^{2}(1+\phi(x))=n_{2}(x)+O\left(\eta^{2}\right) .
$$

